\title{
REGULARITY PROPERTIES OF SOLUTIONS TO TRANSMISSION PROBLEMS
}

\author{
LUIS ESCAURIAZA AND JIN KEUN SEO
}

\begin{abstract}
We show that the gradients of solutions to certain elliptic and parabolic transmission problems with internal Lipschitz boundary and constant coefficients at each side of the internal boundary are square integrable along the internal boundary.
\end{abstract}

\section{INTRODUCTION}

In $[\mathrm{E}, \mathrm{F}, \mathrm{V}]$ we considered a weak solution $u \in W^{1,2}(B)$, the space of square integrable functions on the unit ball, $B=\left\{X \in \mathbf{R}^{n}|| X \mid<1\right\}$ with distributional derivatives in $L^{2}(B)$, of the divergence form operator $L u=$ $\operatorname{div}(A(X) \nabla u(X))=0$, where $A(X)=k \chi_{D}+\chi_{\mathbf{R}^{n} \backslash D}$ and $D$ denotes a Lipschitz domain contained in the ball of radius $1 / 2$ centered at zero, $B_{1 / 2}$ (see the body of the paper for the relevant definitions). There we showed that the gradient of $u$ has a restriction to the boundary of $D, \partial D$, and that it lies in $L^{2}(\partial D)$. In particular, if $N\left(\nabla u^{+}\right)$and $N\left(\nabla u^{-}\right)$denote respectively the nontangential maximal functions of the gradient of $u$ from inside and outside of $D$, there is a constant $C$ depending on the Lipschitz character of $D$ such that

$$
\left\|N\left(\nabla u^{ \pm}\right)\right\|_{L^{2}(\partial D)} \leq C\|u\|_{W^{1,2}(B)} \text {. }
$$

To obtain this result we first showed that the operator $\lambda I-K^{*}$ is invertible on $L^{2}(\partial D)$, where $|\lambda|>1 / 2$, and

$$
K^{*}(f)(P)=\text { p.v. } \frac{1}{\omega_{n}} \int_{\partial D} \frac{\langle P-Q, N(P)\rangle}{|P-Q|^{n}} f(Q) d \sigma(Q) \text { for } f \in L^{2}(\partial D),
$$

where $d \sigma$ and $N(P)$ denote respectively the surface measure on $\partial D$ and the outer unit normal at a point $P$ on $\partial D, \omega_{n}$ the surface area of the unit sphere in $\mathbf{R}^{n}$, and $\langle\cdot, \cdot\rangle$ denotes the scalar product on $\mathbf{R}^{n}$. As a consequence, we obtained a representation of $u$ in a neighborhood of $D$ as the sum of a single layer potential and of a Newtonian potential. In this paper we will obtain similar results for more general equations. In particular, we will show that the same regularity holds when

$$
L u=\operatorname{div}(A(X) \nabla u(X)) \text { with } A(X)=C_{1} \chi_{D}+C_{2} \chi_{\mathbf{R}^{n} \backslash D},
$$

Received by the editors April 24, 1991.

1991 Mathematics Subject Classification. Primary 35B65, 35C15, 35D10, 35E99, 42B20, 45E05.

Key words and phrases. Transmission problems, single layer potential, Lipschitz domains.

The first author was partially supported by NSF grant DMS 90007599 .

The second author was partially supported by NSF grant DMS 8421377-03 and GARC-KOSEF. 
where the matrices $C_{1}$ and $C_{2}$ are constant symmetric positive definite, and $C_{1}-C_{2}$ is positive or negative semidefinite. There are some works where the regularity of solutions to this type of equations is studied [L, R, U], but in them the boundary of $D$ is required to be sufficiently smooth so that the boundary can be flattened. Of course, in this case the regularity of the solution is much better; for instance, when $\partial D$ is locally the graph of a function in the class $W^{p, n}$ and $p>n$ the gradient of $u$ is Hölder continuous up till the boundary on both sides of $D$ (see [L, R, U]).

We will also show that the same type of regularity holds when $\vec{u}=\left(u^{1}, \ldots, u^{n}\right)$ $\in\left[W^{1,2}(B)\right]^{n}$ is a weak solution to a divergence from elliptic system

$$
S^{\alpha} u=D_{i}\left(a_{i j}^{\alpha \beta}(X) D_{j} u^{\beta}\right)=0 \text { for all } \alpha=1, \ldots, n,
$$

where

and

$$
a_{i j}^{\alpha \beta}(X)= \begin{cases}\mu\left(\delta_{\alpha \beta} \delta_{i j}+\delta_{i \beta} \delta_{j \alpha}\right)+\lambda \delta_{j \beta} \delta_{\alpha i} & \text { for } X \in D, \\ \tilde{\mu}\left(\delta_{\alpha \beta} \delta_{i j}+\delta_{i \beta} \delta_{j \alpha}\right)+\tilde{\lambda} \delta_{j \beta} \delta_{\alpha i} & \text { for } X \in \mathbf{R}^{n} \backslash D\end{cases}
$$

$$
\mu, \tilde{\mu}>0, \quad \lambda>-2 \mu / n, \quad \tilde{\lambda}>-2 \tilde{\mu} / n ;
$$

that is, the coefficients coincide with those of two elasticity systems with Lamé constants $\mu, \lambda$, and $\tilde{\mu}$ and $\tilde{\lambda}$ respectively in the interior and exterior of $D$. In this case our method only yields the regularity result when $\mu \leq \tilde{\mu}$ and $\lambda \leq \tilde{\lambda}$ or $\mu \geq \tilde{\mu}$ and $\lambda \geq \tilde{\lambda}$.

The method we use here to obtain the above estimates relies on the RellichNecas indentities [N], and the so called paraproducts which where introduced in $[D, K, V, 1]$ to solve the Dirichlet problem for the biharmonic operator in Lipschitz domains. Though this method gives directly after some technical arguments the above estimates, we have chosen to show first that certain mappings associated to the single layer potentials of the operators at both sides of $D$ are invertible, and as a consequence, as in [E, F, V], to obtain these estimates. In particular, we will show that when $S$ and $\widetilde{S}$ denote respectively the single layer potentials of the constant coefficient operators in the interior and exterior of $D$ in $(0.1)$, the mapping

$$
\begin{gathered}
\quad L^{2}(\partial D) \times L^{2}(\partial D) \rightarrow L_{1}^{2}(\partial D) \times L^{2}(\partial D), \\
(f, g) \rightarrow\left(S(f)-\widetilde{S}(g),\left\langle C_{1} N, \nabla S(f)^{+}\right\rangle-\left\langle C_{2} N, \nabla \widetilde{S}(g)^{-}\right\rangle\right),
\end{gathered}
$$

is invertible, where the superscripts + and - denote respectively the restrictions of the gradients to the boundary of $D$ from the interior and exterior. Analogously, we will show that when $S$ and $\tilde{S}$ denote respectively the single layer potentials of the constant coefficient operators in the interior and exterior of $D$ in (0.2), the operator from $\left[L^{2}(\partial D)\right]^{n} \times\left[L^{2}(\partial D)\right]^{n} \rightarrow\left[L_{1}^{2}(\partial D)\right]^{n} \times\left[L^{2}(\partial D)\right]^{n}$ mapping $(\vec{f}, \vec{g})$ into

$$
\left(S(\vec{f})-\tilde{S}(\vec{g}), \frac{\partial}{\partial v} S(\vec{f})^{+}-\frac{\partial}{\partial \tilde{v}} \tilde{S}(\vec{g})^{-}\right),
$$

where $\partial / \partial v$ and $\partial / \partial \tilde{v}$ denote the conormal operators (so-called tractions) associated respectively to the elasticity systems in (0.2), is also invertible.

We will also show that the same method applies to obtain analogous estimates for solutions to parabolic problems with transmission conditions; that is, if $u \in$ 
$C\left([0, T], L^{2}(B)\right) \cap L^{2}\left([0, T], W^{1,2}(B)\right)$ with $u(X, 0)=0$ is a weak solution to $D_{t} u-\operatorname{div}(A(X, t) \nabla u(X, t))$ on $B \times[0, T]$, with $A(X, t)=C_{1} \chi_{\Omega_{T}}+C_{2} \chi_{\mathrm{R}^{n+1} \backslash \Omega_{T}}$, and where $\Omega_{T}$ is a Lipschitz domain in time and space contained in $B_{1 / 2} \times$ $[0, T]$ whose lateral boundary is not horizontal, and $C_{1}, C_{2}$ satisfy the same conditions as before, then

$$
\begin{gathered}
\left\|N\left(\nabla u^{ \pm}\right)\right\|_{L^{2}\left(\partial \Omega_{T} \cap B \times[0, T]\right)}+\left\|D_{t} u\right\|_{L^{2}\left(B_{3 / 4} \times[0, T]\right)} \\
\leq C\left\{\|u\|_{L^{2}\left([0, T], W^{1 / 2}(B)\right)}+\left\|D_{t} u\right\|_{L^{2}(K)}\right\}
\end{gathered}
$$

where $C$ depends as before on the Lipschitz character of $\Omega_{T}$ and $K$ is a compact subset of $B \times[0, T]$ away from the lateral boundary of $\Omega_{T}$. Here, $L^{2}\left([0, T], W^{1,2}(B)\right)$ denotes the space of functions $u \in L^{2}([0, T] \times B)$ such that $u(\cdot, t)$ lies in $W^{1,2}(B)$ for almost every $t$ in $[0, T]$, and

$$
\|u\|_{L^{2}\left([0, T], W^{1,2}(B)\right)}^{2}=\int_{0}^{T} \int_{B}|u(x, t)|^{2}+|\nabla u(X, t)|^{2} d X d t<\infty .
$$

Throughout this paper we will assume that the dimension $n$ is greater or equal than 3, leaving the details when $n=2$ for the reader.

\section{NotATION AND DEFINITIONS}

The letter $X$ will denote a point in $\mathbf{R}^{n}$ and the letters $P$ and $Q$ points on the boundary of a bounded open domain $D$. Derivatives $\partial / \partial X_{i}$ will often be written $D_{i}$, and time derivatives $D_{t}$. An open ball of radius $r$ centered at a point $X$ will be denoted as $B_{r}(X)$, and if the center of the ball is the origin we will simply use the notation $B_{r}$. In particular $B=B_{1}$.

We will often use the index summation convention of repeated indices, and the action of a matrix $A=\left(a_{i j}\right)$ on a vector $\xi=\left(\xi_{1}, \ldots, \xi_{n}\right)$ is defined as $(A \xi)_{i}=a_{i j} \xi_{j}$ for $i=1, \ldots, n$.

A bounded open connected domain $D$ in $\mathbf{R}^{n}$ is a Lipschitz domain if for each point $P \in \partial D$ there is a coordinate system $(x, s), x \in \mathbf{R}^{n-1}, s \in \mathbf{R}$, so that with respect to this coordinate system $P=(0,0)$, and a double truncated cylinder $Z$ centered at $P$ with axis parallel to the $s$-axis and whose bottom and top are at a positive distance from $\partial D$, and a Lipschitz function $\varphi$ with $\|\nabla \varphi\|_{L^{\infty}\left(\mathbf{R}^{n-1}\right)} \leq m$, so that $Z \cap D=Z \cap\{(x, s) \mid s>\varphi(x)\}$ and $Z \cap \partial D=$ $Z \cap\{(x, s) \mid s=\varphi(x)\}$.

If $u$ is a function defined on a neighborhood of $D$ we define the interior and exterior nontangential maximal functions of $u$ at $P \in \partial D$ as

$$
N\left(u^{+}\right)(P)=\operatorname{Sup}_{X \in \Gamma^{+}(P)}|u(X)| \text { and } N\left(u^{-}\right)(P)=\operatorname{Sup}_{X \in \Gamma^{-}(P)}|u(X)|,
$$

where $\Gamma^{+}(P)=\left\{X \in D / d(X, P) \leq \frac{3}{2} d(X, \partial D)\right.$ and $\left.d(X, \partial D) \leq r\right\}, \Gamma^{-}(P)=$ $\left\{X \in \mathbf{R}^{n} \mid D / d(X, P) \leq \frac{3}{2} d(X, \partial D)\right\}$, where $r$ is chosen so that the above set is strictly contained in the interior of $D$. When $u$ is only defined in the interior of $D$ we define its nontangential maximal function as

$$
N(u)(P)=\operatorname{Sup}_{X \in \Gamma^{+}(P)}|u(X)| .
$$

We say that $f \in L_{1}^{p}(\partial D), 1<p<\infty$, if $f \in L^{p}(\partial D)$ and for every cylinder $Z$ as in the above definition with associated Lipschitz function $\varphi$, there are 
$L^{p}(\partial D \cap Z)$ functions $g_{1}, \ldots, g_{n-1}$ such that

$$
\int_{\mathbf{R}^{n-1}} h(x) g_{j}(x, \varphi(x)) d x=-\int_{\mathbf{R}^{n-1}} \frac{\partial}{\partial x_{j}} h(x) f(x, \varphi(x)) d x
$$

whenever $h \in C_{0}^{\infty}\left(\mathbf{R}^{n-1} \cap Z\right)$.

It is easy to see that given $f \in L_{1}^{p}(\partial D)$ it is possible to define a unique vector $\nabla_{t} f$, at almost every $P \in \partial D$ so that $\left\|\nabla_{t} f\right\|_{L^{p}(\partial D)}$ is equivalent to the sum over all the coordinate cylinders in a given covering of $\partial D$ of the $L^{p}$ norms of the locally defined functions $g_{j}$ for $f$, occurring in the definition of $L_{1}^{p}(\partial D)$. The resulting vector field $\nabla_{t} f$, will be called the tangential gradient of $f$. If $F$ is a function defined on $\mathbf{R}^{n}, \nabla_{t} F$ is orthogonal to the normal vector $N$, and $\nabla F=\nabla_{t} F+\langle N, \nabla F\rangle N$. In local coordinates $\nabla_{t} F$ may be realized as $\left(\left\langle\nabla F, T_{1}\right\rangle, \ldots,\left\langle\nabla F, T_{n-1}\right\rangle\right)$, where $T_{1}, \ldots, T_{n-1}$ are "tangent" vectors to $\partial D$ at $P=(x, \varphi(x))$, given by

$$
T_{j}=\left(0, \ldots, 1, \ldots, 0, \frac{\partial}{\partial x_{j}} \varphi(x)\right)\left[1+|\nabla \varphi(x)|^{2}\right]^{-1 / 2},
$$

where the number 1 is located in the $j$ th coordinate. $L_{1}^{p}(\partial D)$ may be normed as $\|f\|_{L^{p}(\partial D)}+\left\|\nabla_{t} f\right\|_{L^{p}(\partial D)}$ and for $F$ as above we have

$$
\nabla F=\langle\nabla F, N\rangle N+\sum_{j=1}^{n}\left\langle\nabla F, T_{j}\right\rangle T_{j} \quad \text { almost everywhere on } \partial D .
$$

The following formulation of Poincaré's inequality will be of use to us.

Theorem 0. Let $D$ be a Lipschitz domain. Assume $\int_{\partial D} F d \sigma=0$. Then

$$
\int_{\partial D}|F|^{2} d \sigma \leq C \int_{\partial D}\left|\nabla_{t} F\right|^{2} d \sigma
$$

where $C$ depends only on the Lipschitz character of $D$.

Given a constant coefficient elliptic operator

$$
L u=\sum_{i, j=1}^{n} a_{i j} D_{i j} u, \quad \text { with } a_{i j}=a_{j i} \text { for all } i, j=1, \ldots, n,
$$

and satisfying for some $c>0$

$$
c|\xi|^{2} \leq \sum_{i, j=1}^{n} a_{i j} \xi_{i} \xi_{j} \leq c^{-1}|\xi|^{2} \quad \text { for all } \xi \in \mathbf{R}^{n}
$$

we will consider the single layer potential associated to $L$ in the domain $D$, given by

$$
S(f)(X)=\int_{\partial D} \Gamma(X-Q) f(Q) d \sigma(Q) \text { for } X \in \mathbf{R}^{n} \text { and } f \in L^{p}(\partial D),
$$

where $\Gamma(X)$ is the fundamental solution of the operator $L$. It is well known that the following properties are satisfied:

$L S(f)(X)=0$ on $\mathbf{R}^{n} \backslash \partial D$, and $S(f)$ is continuous at almost every $P \in \partial D$ with respect to surface measure $d \sigma$. If

$$
K_{i}(f)(P)=\mathrm{p} . \mathrm{v} \cdot \int_{\partial D} D_{i} \Gamma(P-Q) f(Q) d \sigma(Q),
$$


then

$$
\begin{gathered}
\left\|K_{i}(f)\right\|_{L^{p}(\partial D)} \leq C\|f\|_{L^{p}(\partial D)}, \\
\left\|N\left(\nabla S(f)^{+}\right)\right\|_{L^{p}(\partial D)}+\left\|N\left(\nabla S(f)^{-}\right)\right\|_{L^{p}(\partial D)} \leq C\|f\|_{L^{p}(\partial D)},
\end{gathered}
$$

for all $1<p<+\infty$, where $C$ depends on the Lipschitz character of $D$ and $c$ [C, Mc, Me]. Standard arguments yield the trace formula [F, J, R]

$$
\lim _{\substack{X \in \Gamma^{ \pm}(P) \\ X \rightarrow P}} D_{i} S(f)(X)=\mp \frac{1}{2} \frac{N_{i}(P)}{\langle A N(P), N(P)\rangle} f(P)+K_{i}(f)(P),
$$

where $A$ is the constant coefficient matrix $A=\left(a_{i j}\right)$. In particular, the conormal associated to $L$ has the following trace on $\partial D$ when acting on $S(f)$ :

$$
\lim _{\substack{X \in \Gamma^{ \pm}(P) \\ X \rightarrow P}}\langle A \nabla S(f)(X), N(P)\rangle=\mp \frac{1}{2} f(P)+K(f)(P),
$$

where

$$
K(f)(P)=\text { p.v. } \int_{\partial D}\langle A \nabla \Gamma(P-Q), N(P)\rangle f(Q) d \sigma(Q) \text { for } P \in \partial D .
$$

Also,

$$
\lim _{\substack{X \in \Gamma^{+}(P) \\ X \rightarrow P}}\left\langle\nabla S(f)(X), T_{i}\right\rangle=\lim _{\substack{x \in \Gamma^{-}(P) \\ X \rightarrow P}}\left\langle\nabla S(f)(X), T_{i}\right\rangle \quad \text { for all } 1 \leq i \leq n-1 .
$$

In the case of an elasticity system $\mu \Delta \vec{u}+(\lambda+\mu) \nabla(\operatorname{div} \vec{u})=0$, with Lamé constants $\mu$ and $\lambda, \mu>0$, and $\lambda>-2 \mu / n$, the single layer potential is given by $S(\vec{f})=\left(S(\vec{f})_{1}, \ldots, S(\vec{f})_{n}\right)$, where

$$
S(\vec{f})_{i}(X)=\int_{\partial D} \Gamma^{i j}(X-Q) f_{j}(Q) d \sigma(Q) \text { for } X \in \mathbf{R}^{n}, i=1, \ldots, n,
$$

$\vec{f}=\left(f_{1}, \ldots, f_{n}\right) \in\left[L^{p}(\partial D)\right]^{n}, 1<p<\infty$, and $\left(\Gamma^{i j}(X)\right)$ is the fundamental solution matrix

$$
\Gamma^{i j}(X)=\frac{A}{\omega_{n}(2-n)} \delta_{i j}|X|^{2-n}+\frac{B}{\omega_{n}} X_{i} X_{j}|X|^{-n},
$$

where $A=\frac{1}{2}(1 / \mu+1 /(2 \mu+\lambda))$ and $B=\frac{1}{2}(1 / \mu-1 /(2 \mu+\lambda))$.

As before, the following properties are well known:

$$
\mu \Delta S(\vec{f})+(\mu+\lambda) \nabla(\operatorname{div}(S(\vec{f})))=0
$$

on $\mathbf{R}^{n} \backslash \partial D, S(\vec{f})$ is continuous at almost every $P$ on $\partial D$, and from the results in $[\mathrm{C}, \mathrm{Mc}, \mathrm{Me}]$ we have

$$
\left\|N\left(\nabla S(\vec{f})^{+}\right)\right\|_{L^{p}(\partial D)}+\left\|N\left(\nabla S(\vec{f})^{-}\right)\right\|_{L^{p}(\partial D)} \leq C\|\vec{f}\|_{L^{p}(\partial D)} \quad \text { for } 1<p<\infty,
$$

where $C$ depends on the Lipschitz character of $D, \lambda$, and $\mu$.

As before, standard arguments yield the trace formulas:

$$
\begin{aligned}
\lim _{\substack{X \in \Gamma^{ \pm}(P) \\
X \rightarrow P}} D_{i} S(\vec{f})_{j}(X)= & \pm\left\{\frac{1}{2 \mu} N_{i} f_{j}(P)-B N_{i} N_{j}\langle N, \vec{f}(P)\rangle\right\} \\
& + \text { p.v. } \int_{\partial D} D_{i} \Gamma^{i k}(P-Q) f_{k}(Q) d \sigma(Q) .
\end{aligned}
$$


We will write the matrix $a_{i j}^{\alpha \beta}$ associated to an elasticity system as $a_{i j}^{\alpha \beta}=$ $\mu\left(\delta_{\alpha \beta} \delta_{i j}+\delta_{i \beta} \delta_{j \alpha}\right)+\lambda \delta_{j \beta} \delta_{\alpha i}$, for $\alpha, \beta, i, j=1, \ldots, \nu$; so that the conormal $\partial / \partial v$ associated to the system is given by the so-called traction,

$$
\frac{\partial \vec{u}}{\partial \nu}=\mu\left(\nabla \vec{u}+\nabla \vec{u}^{\perp}\right) N+\lambda \operatorname{div}(\vec{u}) N,
$$

where $\nabla \vec{u}$ denotes the matrix $\left(D_{i} u^{j}\right)$ and $\nabla \vec{u}^{\perp}$ its transpose $\left(D_{j} u^{i}\right)$. In particular,

$$
\lim _{\substack{x \in \Gamma^{ \pm}(P) \\ X \rightarrow P}} \frac{\partial}{\partial \nu} S(\vec{f})(X)= \pm \frac{1}{2} \vec{f}+K(\vec{f})
$$

where $K$ is a bounded singular integral operator on $\left[L^{p}(\partial D)\right]^{n}, 1<p<\infty$.

\section{Case of a Single equation}

In this section we will prove the following theorems.

Theorem 1. Let $u \in W^{1,2}(B)$ be a weak solution to $(0.1)$ on $B$, where the constant matrices $C_{1}$ and $C_{2}$ are symmetric and elliptic, and $C_{1}-C_{2}$ is either positive or negative semidefinite, and $D$ is a Lipschitz domain contained in $B_{1 / 2}$. Then there is a constant $C$ depending only on the Lipschitz character of $D$, the ellipticity constants of $C_{1}$ and $C_{2}$, and the smallest and largest of the absolute value of the nonzero eigenvalues of $C_{1}-C_{2}$ so that $\left\|N\left(\nabla u^{ \pm}\right)\right\|_{L^{2}(\partial D)} \leq$ $C\|u\|_{W^{1,2(B)}}$.

Theorem 2. Let $C_{1}, C_{2}$, and $D$ be as in Theorem 1 , and $S$ and $\widetilde{S}$ denote respectively the single layer potentials of the constant coefficient operators with coefficient matrices $C_{1}$ and $C_{2}$. Then the mapping

$$
\begin{gathered}
L^{2}(\partial D) \times L^{2}(\partial D) \rightarrow L_{1}^{2}(\partial D) \times L^{2}(\partial D), \\
(f, g) \rightarrow\left(S(f)-\widetilde{S}(g),\left\langle C_{1} N, \nabla S(f)^{+}\right\rangle-\left\langle C_{2} N, \nabla \widetilde{S}(g)^{-}\right\rangle\right),
\end{gathered}
$$

is an invertible operator.

We will first show how to prove the first theorem.

Proof of Theorem 1. Let $u$ be as in the statement of Theorem 1, $\varphi \in C_{0}^{\infty}\left(\mathbf{R}^{n}\right)$ with $\varphi=1$ on $B_{3 / 4}$, and $\varphi=0$ outside $B_{7 / 8}$. If $C_{2}=\left(c_{i j}\right)$ and we define $h=\sum_{i, j=1}^{n} a_{i j} D_{i j}(u \varphi) \chi_{\mathbf{R}^{n} \backslash D}$, we have $\|h\|_{L^{2}\left(\mathbf{R}^{n}\right)} \leq C\|u\|_{W^{1,2}(B)}$. Setting $w=$ $u \varphi-\Gamma(h)+\widetilde{S}(g)$ for $X \in \mathbf{R}^{n} \backslash D$ and $w=u+S(f)$ for $X \in D$, where $S$ and $\widetilde{S}$ are as in Theorem 2, and $\Gamma(h)$ denotes the Newtonian potential of $h$ associated to the constant coefficient operator outside $D$, one can show as in [E, F, V, Theorem 1] that for any $f$ and $g \in L^{2}(\partial D)$ the function $w$ satisfies for all $\phi \in C_{0}^{\infty}\left(\mathbf{R}^{n}\right)$

$$
\begin{aligned}
& \int_{D}\left\langle C_{1} \nabla w, \nabla \phi\right\rangle d X+\int_{\mathbf{R}^{n} \backslash D}\left\langle C_{2} \nabla w, \nabla \phi\right\rangle d X \\
& \quad=\int_{\partial D}\left\{\left\langle C_{1} N, \nabla S(f)^{+}\right\rangle-\left\langle C_{2} N, \nabla \widetilde{S}(g)^{-}\right\rangle-\left\langle C_{2} N, \nabla \Gamma(h)\right\rangle\right\} \phi d \sigma .
\end{aligned}
$$

From Theorem 2 we can find $f$ and $g$ in $L^{2}(\partial D)$ so that $S(f)-\tilde{S}(g)=-\Gamma(h)$ and $\left\langle C_{1} N, \nabla S(f)^{+}\right\rangle-\left\langle C_{2} N, \nabla \widetilde{S}(g)^{-}\right\rangle=-\left\langle C_{2} N, \nabla \Gamma(h)\right\rangle$ on $\partial D$. With this 
choice of $f$ and $g$ the function $w$ lies in $W_{\text {loc }}^{1,2}\left(\mathbf{R}^{n}\right), w(X)=O\left(|X|^{2-n}\right)$ at infinity, and satisfies

$$
\begin{aligned}
& \int_{D}\left\langle C_{1} \nabla w(X), \nabla \phi(X)\right\rangle d X \\
& \quad+\int_{\mathbf{R}^{n} \backslash D}\left\langle C_{2} \nabla w(X), \nabla \phi(X)\right\rangle d X=0 \quad \text { for all } \phi \in C_{0}^{\infty}\left(\mathbf{R}^{n}\right) .
\end{aligned}
$$

These and the maximum principle for weak solutions to divergence form elliptic equations imply that $w$ is identically zero. Therefore, we obtain a representation formula for $u$ in a neighborhood of $D$, which combined with Theorem 2 , (1.1), and the estimate $\|\nabla \Gamma(h)\|_{L^{\infty}\left(B_{3 / 4}\right)} \leq C\|u\|_{W^{1,2}(B)}$ implies the theorem.

Proof of Theorem 2. The operator in Theorem 2 is one-to-one. To see this observe that if $f$ and $g \in L^{2}(\partial D)$, and $S(f)-\widetilde{S}(g)=0,\left\langle C_{1} N, \nabla S(f)^{+}\right\rangle-$ $\left\langle C_{2} N, \nabla \tilde{S}(g)^{-}\right\rangle=0$ on $\partial D$, the function $u$ defined as $u=S(f)$ on $D$ and $u=\widetilde{S}(g)$ on $\mathbf{R}^{n} \backslash D$ lies in $W_{\text {loc }}^{1,2}\left(\mathbf{R}^{n}\right)$ with $u(X)=O\left(|X|^{2-n}\right)$ at infinity, and is a weak solution on $\mathbf{R}^{n}$ to $L u=0$ with $L$ as in $(0.1)$. Thus, $u$ must be identically zero. On the other hand, the identities

$$
\begin{gathered}
\int_{\mathbf{R}^{n} \backslash D}\left\langle C_{1} \nabla S(f), \nabla S(f)\right\rangle d X=-\int_{\partial D} S(f)\left\langle C_{1} N, \nabla S(f)^{+}\right\rangle d \sigma, \\
\int_{D}\left\langle C_{2} \nabla \tilde{S}(g), \nabla \tilde{S}(g)\right\rangle d X=\int_{\partial D} \tilde{S}(g)\left\langle C_{2} N, \nabla \tilde{S}(g)^{-}\right\rangle d \sigma
\end{gathered}
$$

show that $S(f)$ and $\widetilde{S}(g)$ are identically zero on $\mathbf{R}^{n}$, and from the jumps on $\partial D$ of the conormals derivatives of $S(f)$ and $\tilde{S}(g)$ (see (1.2) and (1.3)) we get $f=g=0$ on $\partial D$.

Next, we will show that the following estimate holds:

$$
\begin{aligned}
& \|f\|_{L^{2}(\partial D)}+\|g\|_{L^{2}(\partial D)} \\
& \quad \leq C\left\{\|S(f)-\widetilde{S}(g)\|_{L_{1}^{2}(\partial D)}+\left\|\left\langle C_{1} N, \nabla S(f)^{+}\right\rangle-\left\langle C_{2} N, \nabla \tilde{S}(g)^{-}\right\rangle\right\|_{L^{2}(\partial D)}\right. \\
& \left.+\left|\int_{\partial D} S(f) d \sigma\right|+\left|\int_{\partial D} \tilde{S}(g) d \sigma\right|\right\},
\end{aligned}
$$

where $C$ is as in Theorem 1. To prove (2.1) we set $u^{+}=S(f)$ on $D$ and $u^{-}=\tilde{S}(g)$ on $\mathbf{R}^{n} \backslash D$, and observe that the function $u$ defined on $\mathbf{R}^{n}$ as $u^{+}$and $u^{-}$respectively in the interior and exterior of $D$, satisfies for all $\phi \in C_{0}^{\infty}\left(\mathbf{R}^{n}\right)$

$$
\begin{gathered}
\int_{D}\left\langle C_{1} \nabla u, \nabla \phi\right\rangle d X+\int_{\mathbf{R}^{n} \backslash D}\left\langle C_{2} \nabla u, \nabla \phi\right\rangle d X \\
=\int_{\partial D}\left\{\left\langle C_{1} N, \nabla S(f)^{+}\right\rangle-\left\langle C_{2} N, \nabla \tilde{S}(g)^{-}\right\rangle\right\} \phi d \sigma, \\
u^{+}-u^{-}=S(f)-\tilde{S}(g) \text { on } \partial D .
\end{gathered}
$$

After a linear change of coordinates and abusing the notation we may assume that $C_{1}=A$ and $C_{2}=I$, where $I$ denotes the identity matrix, and $A$ is a 
diagonal matrix with diagonal elements $\alpha_{1}, \alpha_{2}, \ldots$, and $\alpha_{n}$, so that now $u$ satisfies for all $\phi \in C_{0}^{\infty}\left(\mathbf{R}^{n}\right)$

$$
\begin{gathered}
\int_{D}\langle A \nabla u, \nabla \phi\rangle d X+\int_{\mathbf{R}^{n} \backslash D}\langle\nabla u, \nabla \phi\rangle d X \\
=\int_{\partial D}\left\{\left\langle A N, \nabla u^{+}\right\rangle-\left\langle N, \nabla u^{-}\right\rangle\right\} \phi d \sigma, \\
u^{+}-u^{-}=h_{1} \text { and }\left\langle A N, \nabla u^{+}\right\rangle-\left\langle N, \nabla u^{-}\right\rangle=h_{2} \quad \text { on } \partial D,
\end{gathered}
$$

where $h_{1} \in L_{1}^{2}(\partial D)$ and $h_{2} \in L^{2}(\partial D)$. It is easy to check that under the change of coordinates, the assumption that $C_{1}-C_{2}$ is positive or negative semidefinite is preserved, and becomes $\alpha_{i}-1 \leq 0$ for all $i=1, \ldots, n$ or $\alpha_{i}-1 \geq 0$ for all $i=1, \ldots, n$. In this context we will show the following inequality:

$$
\begin{aligned}
& \left\|\nabla u^{+}\right\|_{L^{2}(\partial D)}+\left\|\nabla u^{-}\right\|_{L^{2}(\partial D)} \\
& \quad \leq C\left\{\left\|h_{1}\right\|_{L_{1}^{2}(\partial D)}+\left\|h_{2}\right\|_{L^{2}(\partial D)}+\left\|\nabla u^{+}\right\|_{L^{2}(D)}+\left\|\nabla u^{-}\right\|_{L^{2}(B \backslash D)}\right\},
\end{aligned}
$$

where $C$ depends on the Lipschitz character of $D$ and the smallest of $\left|\alpha_{i}-1\right|$ with $\alpha_{i}-1 \neq 0$. Returning to the original coordinates and recalling that

$$
\int_{D}\left\langle C_{1} \nabla S(f), \nabla S(f)\right\rangle d X=\int_{\partial D} S(f)\left\langle C_{1} N, \nabla S(f)^{+}\right\rangle d \sigma
$$

and

$$
\int_{\mathbf{R}^{n} \backslash D}\left\langle C_{2} \nabla \widetilde{S}(g), \nabla \widetilde{S}(g)\right\rangle d X=-\int_{\partial D} \widetilde{S}(g)\left\langle C_{2} N, \nabla \widetilde{S}(g)^{-}\right\rangle d \sigma,
$$

we will get from (2.3) and Theorem 0 that

$$
\begin{aligned}
& \left\|\nabla S(f)^{+}\right\|_{L^{2}(\partial D)}+\left\|\nabla \tilde{S}(g)^{-}\right\|_{L^{2}(\partial D)} \\
& \leq C\left\{\|S(f)-\widetilde{S}(g)\|_{L_{1}^{2}(\partial D)}+\|\left\langle C_{1} N, \nabla S(f)^{+}\right\rangle\right. \\
& \left.\quad-\left\langle C_{2} N, \nabla \widetilde{S}(g)^{-}\right\rangle \|_{L^{2}(\partial D)}+\left|\int_{\partial D} S(f) d \sigma\right|+\left|\int_{\partial D} \tilde{S}(g) d \sigma\right|\right\} .
\end{aligned}
$$

Moreover, from the results in [V] and by a simple linear change of coordinates there is a constant $C$ depending only on the Lipschitz character of $D$ and the ellipticity constants of the matrices $C_{1}$ and $C_{2}$, so that for $f \in L^{2}(\partial D)$ the following holds:

$$
\begin{aligned}
& \|f\|_{L^{2}(\partial D)} \leq C\left\{\left\|\nabla S(f)^{+}\right\|_{L^{2}(\partial D)}+\left|\int_{\partial D} S(f) d \sigma\right|\right\}, \\
& \|g\|_{L^{2}(\partial D)} \leq C\left\{\left\|\nabla \widetilde{S}(g)^{-}\right\|_{L^{2}(\partial D)}+\left|\int_{\partial D} \tilde{S}(g) d \sigma\right|\right\},
\end{aligned}
$$

and from the last three inequalities we get (2.1).

To prove (2.3) we recall that there is a vector field $\vec{\beta} \in C_{0}^{\infty}\left(B_{3 / 4}\right)$ such that $\langle\vec{\beta}, N\rangle \geq C$ with $C$ depending only on the Lipschitz character of $D[\mathrm{~V}]$, and the following Rellich-Necas [N] identities:

$$
\begin{aligned}
& \operatorname{div}\left(\vec{\beta}\left\langle A \nabla u^{+}, \nabla u^{+}\right\rangle\right)=2 \operatorname{div}\left(\left\langle\vec{\beta}, \nabla u^{+}\right\rangle A \nabla u^{+}\right)+O\left(\left|\nabla u^{+}\right|^{2}\right) \text { on } D, \\
& \operatorname{div}\left(\vec{\beta}\left\langle\nabla u^{-}, \nabla u^{-}\right\rangle\right)=2 \operatorname{div}\left(\left\langle\vec{\beta}, \nabla u^{-}\right\rangle \nabla u^{-}\right)+O\left(\left|\nabla u^{-}\right|^{2}\right) \quad \text { on } \mathbf{R}^{n} \backslash D \text {. }
\end{aligned}
$$


Integrating on $D$ the first identity we get

$$
\int_{\partial D}\langle\vec{\beta}, N\rangle\left\langle A \nabla u^{+}, \nabla u^{+}\right\rangle d \sigma=2 \int_{\partial D}\left\langle\vec{\beta}, \nabla u^{+}\right\rangle\left\langle A \nabla u^{+}, N\right\rangle d \sigma+O\left(\left\|\nabla u^{+}\right\|_{L^{2}(D)}^{2}\right) \text {. }
$$

Since

$$
\left\langle A \nabla u^{+}, \nabla u^{+}\right\rangle=\left\langle A \nabla u^{+}, T_{l}\right\rangle\left\langle\nabla u^{+}, T_{l}\right\rangle+\left\langle A \nabla u^{+}, N\right\rangle\left\langle\nabla u^{+}, N\right\rangle
$$

and

$$
\left\langle\vec{\beta}, \nabla u^{+}\right\rangle=\langle\vec{\beta}, N\rangle\left\langle\nabla u^{+}, N\right\rangle+\left\langle\vec{\beta}, T_{l}\right\rangle\left\langle\nabla u^{+}, T_{l}\right\rangle,
$$

we obtain from the above integral equality the following formula:

$$
\begin{gathered}
\int_{\partial D}\langle\vec{\beta}, N\rangle\left\{\left\langle A \nabla u^{+}, T_{l}\right\rangle\left\langle\nabla u^{+}, T_{l}\right\rangle-\left\langle A \nabla u^{+}, N\right\rangle\left\langle\nabla u^{+}, N\right\rangle\right\} d \sigma \\
\quad=2 \int_{\partial D}\left\langle\vec{\beta}, T_{l}\right\rangle\left\langle\nabla u^{+}, T_{l}\right\rangle\left\langle A \nabla u^{+}, N\right\rangle d \sigma+O\left(\left\|\nabla u^{+}\right\|_{L^{2}(D)}^{2}\right) .
\end{gathered}
$$

Similarly we get for $u^{-}$the following formula:

$$
\begin{aligned}
& \int_{\partial D}\langle\vec{\beta}, N\rangle\left\{\left\langle\nabla u^{-}, T_{l}\right\rangle\left\langle\nabla u^{-}, T_{l}\right\rangle-\left\langle\nabla u^{-}, N\right\rangle\left\langle\nabla u^{-}, N\right\rangle\right\} d \sigma \\
& \quad=2 \int_{\partial D}\left\langle\vec{\beta}, T_{l}\right\rangle\left\langle\nabla u^{-}, T_{l}\right\rangle\left\langle\nabla u^{-}, N\right\rangle d \sigma+O\left(\left\|\nabla u^{-}\right\|_{L^{2}(B \backslash D)}^{2}\right) .
\end{aligned}
$$

From the transmission conditions (2.3) we can rewrite the above formula in terms of the gradient of $u^{+}, h_{1}$, and $h_{2}$ on $\partial D$, obtaining

$$
\begin{aligned}
\int_{\partial D}\langle\vec{\beta}, N\rangle\left\{\left\langle\nabla u^{+}, T_{l}\right\rangle\left\langle\nabla u^{+}, T_{l}\right\rangle-\left\langle A \nabla u^{+}, N\right\rangle\left\langle A \nabla u^{+}, N\right\rangle\right\} d \sigma \\
=2 \int_{\partial D}\left\langle\vec{\beta}, T_{l}\right\rangle\left\langle\nabla u^{+}, T_{l}\right\rangle\left\langle A \nabla u^{+}, N\right\rangle d \sigma \\
\quad+O\left(\left\|\nabla u^{-}\right\|_{L^{2}(B \backslash D)}^{2}+\left\|\nabla u^{+}\right\|_{L^{2}(\partial D)}\left\{\left\|h_{1}\right\|_{L_{l}^{2}(\partial D)}+\left\|h_{2}\right\|_{L^{2}(\partial D)}\right\}\right) .
\end{aligned}
$$

Subtracting (2.5) from (2.4) we have

$$
\begin{aligned}
\int_{\partial D}\langle\vec{\beta}, N\rangle\left\langle(A-I) \nabla u^{+}, T_{l}\right\rangle\left\langle\nabla u^{+}, T_{l}\right\rangle d \sigma \\
\quad+\int_{\partial D}\langle\vec{\beta}, N\rangle\left\langle(A-I) \nabla u^{+}, N\right\rangle\left\langle A \nabla u^{+}, N\right\rangle d \sigma \\
=O\left(\left\|\nabla u^{-}\right\|_{L^{2}(B \backslash D)}^{2}+\left\|\nabla u^{+}\right\|_{L^{2}(D)}^{2}+\left\|\nabla u^{+}\right\|_{L^{2}(\partial D)}\left\{\left\|h_{1}\right\|_{L_{1}^{2}(\partial D)}+\left\|h_{2}\right\|_{L^{2}(\partial D)}\right\}\right) .
\end{aligned}
$$

From the orthonormality of the linear base $\left\{N, T_{i}, \ldots, T_{n}\right\}$ we have

$$
\begin{aligned}
& \left\langle(A-I) \nabla u^{+}, T_{l}\right\rangle\left\langle\nabla u^{+}, T_{l}\right\rangle+\left\langle(A-I) \nabla u^{+}, N\right\rangle\left\langle A \nabla u^{+}, N\right\rangle \\
& =\left\langle(A-I) \nabla u^{+}, A \nabla u^{+}\right\rangle-\sum_{l=1}^{n}\left\langle(A-I) \nabla u^{+}, T_{l}\right\rangle^{2} \\
& =\left\langle(A-I) \nabla u^{+}, \nabla u^{+}\right\rangle+\left\langle(A-I) \nabla u^{+}, N\right\rangle^{2} .
\end{aligned}
$$

From the above equalities, (2.6), and the transmission conditions (2.2), we get (2.3) when $A-I$ is positive or negative definite. When $A-I$ is only semidefinite 
we only obtain control on some of the partial derivatives of $u^{+}$on $\partial D$. In particular, if $H=\left\{i \mid \alpha_{i}-1 \neq 0\right\}$ we get

$$
\begin{aligned}
\sum_{i \in H}\left\|D_{i} u^{+}\right\|_{L^{2}(D)}^{2} \leq C\left\{\left\|\nabla u^{-}\right\|_{L^{2}(B \backslash D)}^{2}+\left\|\nabla u^{+}\right\|_{L^{2}(D)}^{2}\right. \\
+\left\|\nabla \cdot u^{+}\right\|_{L^{2}(\partial D)}\left\{\left(\left\|h_{1}\right\|_{L_{1}^{2}(\partial D)}+\left\|h_{2}\right\|_{L^{2}(\partial D)}\right)\right\} .
\end{aligned}
$$

But (2.2) can be formally rewritten in the following way

$$
\begin{aligned}
& \int_{\mathbf{R}^{n}}\langle\nabla u, \nabla \phi\rangle d X=-\sum_{i \in H} \int_{D}\left(1-\alpha_{i}\right) D_{i}^{2} u^{+} \phi d X \\
& \quad+\int_{\partial D}\left\{h_{2}+\sum_{i \in H}\left(1-\alpha_{i}\right) D_{i} u^{+} N_{i}\right\} \phi d \sigma \quad \text { for all } \phi \in C_{0}^{\infty}\left(\mathbf{R}^{n}\right), \\
& u^{+}-u^{-}=h_{1}, \quad \text { and }\left\langle A N, \nabla u^{+}\right\rangle-\left\langle N, \nabla u^{-}\right\rangle=h_{2} \quad \text { on } \partial D .
\end{aligned}
$$

Let $\Gamma(X)$ and $\widetilde{S}$ denote respectively the fundamental solution and the single layer potential for Laplace's operator in $D$, and $w$ be the solution to $\Delta w=0$ on $D$ with $w=h_{1}$ on $\partial D$. We define the function

$$
\begin{aligned}
v= & w+\Gamma\left(\sum_{i \in H}\left(1-\alpha_{i}\right) D_{i}^{2} u^{+} \chi_{D}\right) \\
& -\widetilde{S}\left(h_{2}+\sum_{i \in H}\left(1-\alpha_{i}\right) D_{i} u^{+} N_{i}-\langle\nabla w, N\rangle\right) \text { on } D,
\end{aligned}
$$

and

$$
\begin{aligned}
v= & \Gamma\left(\sum_{i \in H}\left(1-\alpha_{i}\right) D_{i}^{2} u^{+} \chi_{D}\right) \\
& -\widetilde{S}\left(h_{2}+\sum_{i \in H}\left(1-\alpha_{i}\right) D_{i} u^{+} N_{i}-\langle\nabla w, N\rangle\right) \text { on } \mathbf{R}^{n} \backslash D .
\end{aligned}
$$

At this point we will recall the following result which is essentially proved in $[\mathrm{K}, \mathrm{V}, \mathrm{D}, 1]$ and whose proof we postpone to the end of this section.

Lemma 1. Let $u$ satisfy $A u=0$ in the interior of a bounded Lipschitz domain $D$, where $A$ is a constant coefficient second order elliptic operator, and $K$ be a smooth homogeneous even function of order $2-n$; i.e., $K(\lambda X)=\lambda^{2-n} K(X)$ and $K(X)=K(-X)$ for all $\lambda>0$ and $X \in \mathbf{R}^{n}$, and set

$$
H(X)=\int_{D} K(X-Y) D_{i} u(Y) d Y \text { for } X \in \mathbf{R}^{n} \text { for some } 1 \leq i \leq n .
$$

Then, there is a constant $C$ depending on the Lipschitz character of $D$ and the ellipticity constants of $A$ such that $\left\|N\left(\nabla H^{ \pm}\right)\right\|_{L^{2}(\partial D)} \leq C\|u\|_{L^{2}(\partial D)}$. Moreover, $\nabla H^{+}=\nabla H^{-}$almost everywhere on $\partial D$, and $H(X)=O\left(|X|^{2-n}\right)$ at infinity.

From Lemma 1 and the well-known estimate, $\|N(\nabla w)\|_{L^{2}(\partial D)} \leq C\left\|h_{1}\right\|_{L_{1}^{2}(\partial D)}$ [J, K, 1], we conclude that $v(X)-u(X)=O\left(|X|^{2-n}\right)$ at infinity. On the other hand, it follows from (2.9) and (1.2) that $v-u$ is harmonic on $\mathbf{R}^{n}$. Hence, 
$u=v$ on $\mathbf{R}^{n}$. From this representation formula for $u$, Lemma 1, and the above estimate we obtain

$\left\|\nabla u^{+}\right\|_{L^{2}(\partial D)}+\left\|\nabla u^{-}\right\|_{L^{2}(\partial D)} \leq C\left\{\left\|h_{1}\right\|_{L_{1}^{2}(\partial D)}+\left\|h_{2}\right\|_{L^{2}(\partial D)}+\sum_{i \in H}\left\|D_{i} u^{+}\right\|_{L^{2}(\partial D)}\right\}$,

which together with (2.8) implies inequality (2.3) as we wanted.

It is easy to show by means of a compactness argument that the estimate (2.1) implies that the operator in Theorem 2 has closed range (see [V]). As in [V], approximation of $D$ by smooth domains and the estimate (2.1) imply that the operator in Theorem 2 has dense range, provided it is known that the range of this operator is dense when $D$ is a smooth domain. But, it is an easy exercise to show using the classical variational methods and Sobolev imbedding that there is a constant $C$ depending only on the Lipschitz character of $D$, such that when given $h_{1}$ and $h_{2}$ in $C_{0}^{\infty}\left(\mathbf{R}^{n}\right)$, there exists a function $u$ satisfying $\operatorname{div}\left(\left(C_{1} \chi_{D}+C_{2} \chi_{\mathbf{R}^{n} \backslash D}\right) \nabla u\right)=0$ on $\mathbf{R}^{n} \backslash \partial D, u^{+}-u^{-}=h_{1}$ in the sense of trace operators, $\left\langle C_{1} N, \nabla u^{+}\right\rangle-\left\langle C_{2} N, \nabla u^{-}\right\rangle=h_{2}$ on $\partial D$, and so that

$$
\|u\|_{L^{q}\left(\mathbf{R}^{n}\right)}+\|\nabla u\|_{L^{2}\left(\mathbf{R}^{n}\right)} \leq C\left\{\left\|\nabla h_{1}\right\|_{L^{2}\left(\mathbf{R}^{n}\right)}+\left\|h_{2}\right\|_{L^{2}(\partial D)}\right\},
$$

where $q=2 n /(n-2)$. To see this one could find for each $r>1$ the weak solution $w_{r} \in W_{0}^{1,2}\left(B_{r}\right)$; the closure of $C_{0}^{\infty}\left(B_{r}\right)$ in $W^{1,2}\left(B_{r}\right)$, to

$$
\operatorname{div}\left(\left(C_{1} \chi_{D}+C_{2} \chi_{\mathbf{R}^{n} \backslash D}\right) \nabla w_{r}\right)=-\operatorname{div}\left(\left(C_{1} \chi_{D}+C_{2} \chi_{\mathbf{R}^{n} \backslash D}\right) \nabla h_{1}\right)+F,
$$

where $F$ acts on test functions as $F(\phi)=\int_{\partial D} \phi h_{2} d \sigma$. Since the estimate (2.10) holds uniformly when $u$ is replaced by $w_{r}$, we have that $w_{r}$ converges to a function $w$, and setting $u^{+}=w+h_{1}$ on $D, u^{-}=w$ on the complement, one constructs such a $u$. Moreover, from the arguments in $[\mathrm{L}, \mathrm{R}, \mathrm{U}]$ and when $D$ is smooth, it follows that the gradient of this function $u$ at both sides of $D$ is a bounded continuous function. Also, Theorem 2 in $[\mathrm{S}, \mathrm{W}]$ shows that $u(X)=O\left(|X|^{2-n}\right)$ at infinity. From all these facts, it follows after a linear change of coordinates (see [V]) that $u$ can be represented inside and outside of $D$ in terms of some densities with respect to the single layer potentials associated to the operators inside and outside of $D$ respectively. From all these we conclude that the range of the operator in Theorem 2 is dense when $D$ is smooth.

We will now indicate how to proceed to prove Lemma 1. By a simple linear change of coordinates and again abusing the notation we may assume that $u$ is harmonic in $D$. After this change of coordinates the function $K$ will turn into a new potential with the same homogeneity. Also, without loss of generality we might assume that the gradient of $u$ is square integrable in $D$, so that the integral defining $H$ is absolutely convergent. In this case it is obvious that $\nabla H^{+}=\nabla H^{-}$almost everywhere on $\partial D$. In [D, $\mathrm{K}, \mathrm{V}, 1$, Lemma $\left.1.3,1.4\right]$ Lemma 1 is proved when $K(X)$ is the fundamental solution of the Laplace operator, but an analysis of the proof of the first lemma which we mentioned in the above reference, shows that the same proof can be carried over when substituting this particular potential by an arbitrary potential $K$ satisfying the conditions in Lemma 1. The only minor difference in the proof of the generalization of this result is that instead of solving $\Delta B(X)=|X|^{2-n}$ on $\mathbf{R}^{n}$, as in the proof of Lemma 1.3 in [D, K, V, 1], we would have to solve $\Delta B(X)=K(X)$, 
observe that $B$ is a smooth homogeneous even function of order $4-n$, so that from the results in $[\mathrm{C}, \mathrm{Mc}, \mathrm{Me}]$ and the method of rotations $[\mathrm{C}, \mathrm{Z}]$, the principal value operators

$$
\text { p.v. } \int_{\partial D} D_{i j k}^{3} B(P-Q) f(Q) d \sigma \quad \text { for all } i, j, k \in\{1, \ldots, n\}
$$

define bounded operators on $L^{2}(\partial D)$; and repeat the same integration by parts argument which is done in their proof. That $\nabla H^{+}=\nabla H^{-}$almost everywhere on $\partial D$ follows from standard arguments and the fact that this is the case when the gradient of $u$ is square integrable in $D$. The last statement in the lemma is a consequence of the representation of $H$ which is obtained when repeating the argument in [D, K, V, 1, Lemma 1.3].

Remark 2.1. We would like to observe that when the argument given to obtain the estimate (2.3) is localized one obtains the following estimate, if $P$ lies on $\partial D, r>0$, and $\Delta_{r}(P)=B_{r}(P) \cap \partial D$ then

$$
\begin{aligned}
& \left\|\nabla u^{+}\right\|_{L^{2}\left(\Delta_{r}(P)\right)}+\left\|\nabla u^{-}\right\|_{L^{2}\left(\Delta_{r}(P)\right)} \\
& \quad \leq C\left\{\left\|\nabla_{t} h_{1}\right\|_{L^{2}\left(\Delta_{r}(P)\right)}+\left\|h_{2}\right\|_{L^{2}\left(\Delta_{r}(P)\right)}+\|\nabla u\|_{L^{2}\left(B_{r}(P)\right)}+\|\nabla u\|_{L^{2}\left(\partial B_{r}(P)\right)}\right\} .
\end{aligned}
$$

Well-known arguments (see [K] and [D, K, V, 1, Theorem 4.1]), and the above estimate show that given a Lipschitz domain $D$, there exists $\varepsilon=\varepsilon(D)$, so that our results extend in the obvious way to $h_{1} \in L_{1}^{p}(\partial D)$ and $h_{2} \in L^{p}(\partial D)$, $2-\varepsilon \leq p \leq 2+\varepsilon$. So that under the same conditions as in Theorem 2 we obtain that this operator is invertible from $L^{p}(\partial D) \times L^{p}(\partial D)$ onto $L_{1}^{p}(\partial D) \times$ $L^{p}(\partial D)$. In particular, this and the argument in Theorem 1 show that under the conditions of this theorem a solution $u$ to a transmission problem satisfies $\left\|N\left(\nabla u^{ \pm}\right)\right\|_{L^{2+\varepsilon}(\partial D)} \leq C\|u\|_{W^{1,2}(B)}$ with $\varepsilon=\varepsilon(D)$.

Remark 2.2. The reader will observe that the method we used in the proof of Theorem 2 can be carried out when the matrices at both sides of $D$ are given by two symmetric elliptic nonconstant matrices $C_{1}(X)$ and $C_{2}(X)$, satisfying that $C_{1}(X)-C_{2}(X)$ is positive definite on $\partial D$, and whose gradients are uniformly bounded on $D$ and $B \backslash D$ respectively. This and the techniques in [J, $K, 1,2]$ show that the same type of regularity can be obtained for solutions to transmission problems with coefficient matrices as above and internal Lipschitz boundary.

Remark 2.3. Finally, we would like to point out that the method we used to obtain the above estimates does not work in all the situations. For instance, when trying to extend the above estimates for arbitrary constant matrices at both sides of $D$, one could think of trying to choose different vector fields $\vec{\beta}$ at each side of $D$, say $\vec{\beta}^{+}$and $\vec{\beta}^{-}$, so that when applying the Rellich-Necas identities to $u^{+}$and $u^{-}$, and rewriting the identity corresponding to $u^{-}$and $\vec{\beta}^{-}$in terms of $u^{+}$, and subtracting this new formula from the original RellichNecas identity corresponding to $\mathcal{u}^{+}$and $\vec{\beta}^{+}$, then one could choose suitable boundary values for $\vec{\beta}^{+}$and $\vec{\beta}^{-}$so that the formula obtained in this way would yield an $L^{2}$ estimate for $\nabla u^{+}$on $\partial D$. But even in the simple case when $\partial D$ is a line segment and $n=2$, the reader will easily verify that if $C_{1}$ is a diagonal matrix with entries $\alpha$ and $1 / \alpha$, where $\alpha>0$, and $C_{2}$ is the identity matrix, 
then there is no choice of $\vec{\beta}^{+}$and $\vec{\beta}^{-}$which would give control on the $L^{2}$ norm of $\nabla u^{+}$along this line segment. Essentially, this is due to the fact that in this case when the Rellich-Necas identity for $u^{-}$is written in terms of $u^{+}$, one obtains a multiple of the original Rellich-Necas identity for $u^{+}$.

\section{CASE OF TRANSMISSION FOR ELASTICITY}

In this section we will show that the methods used before apply to obtain similar results for solutions to transmission problems associated to some systems. In particular we consider solutions $\vec{u} \in\left[W^{1,2}(B)\right]^{n}$ to $S \vec{u}=0$ on $B$, where $S$ is as in (0.2). That is, $\vec{u}$ satisfies for all $\vec{\phi}=\left(\phi^{1}, \ldots, \phi^{n}\right) \in\left[C_{0}^{\infty}\left(\mathbf{R}^{n}\right)\right]^{n}$

$$
\begin{aligned}
\int_{D} \mu & \left(\nabla \vec{u}+\nabla \vec{u}^{\perp}\right) \nabla \vec{\phi}+\lambda \operatorname{div}(\vec{u}) \operatorname{div}(\vec{\phi}) d X \\
& +\int_{B \backslash D} \tilde{\mu}\left(\nabla \vec{u}+\nabla \vec{u}^{\perp}\right) \nabla \vec{\phi}+\tilde{\lambda} \operatorname{div}(\vec{u}) \operatorname{div}(\vec{\phi}) d X=0,
\end{aligned}
$$

where $\left(\nabla \vec{u}+\nabla \vec{u}^{\perp}\right) \nabla \vec{\phi}=\left(D_{i} u^{j}+D_{j} u^{i}\right) D_{i} \phi^{j}$, and where $(0.3)$ is satisfied.

We recall that a system of elasticity with Lamé constants $\mu$ and $\lambda$ satisfying (0.3), and whose coefficient matrix is written as $\mu\left(\delta_{\alpha \beta} \delta_{i j}+\delta_{i \beta} \delta_{j \alpha}\right)+\lambda \delta_{j \beta} \delta_{\alpha i}$ is the standard example of a linear system satisfying the Legendre-Hadamard condition; i.e., $a_{i j}^{\alpha \beta}(X)=a_{j i}^{\beta \alpha}(X)$ and $C|\xi|^{2}|\eta|^{2} \leq a_{i j}^{\alpha \beta}(X) \xi_{i} \xi_{j} \eta^{\alpha} \eta^{\beta}$ for some constant $C$, and for all $X, \xi$, and $\eta$ in $\mathbf{R}^{n}$. From condition (0.3), we also have that the matrix $a_{i j}^{\alpha \beta}(X)$ in (0.2) satisfies the Legendre-Hadamard condition on $\mathbf{R}^{n}$. Also, there is a constant $C$ depending on $\mu, \lambda, \tilde{\mu}$, and $\tilde{\lambda}[\mathrm{D}, \mathrm{K}, \mathrm{V}, 2$, Theorem 1.12] such that

$$
C^{-1}\left|\nabla \vec{v}+\nabla \vec{v}^{\perp}\right|^{2} \leq a_{i j}^{\alpha \beta}(X) D_{j} v^{\beta} D_{i} v^{\alpha} \leq C\left|\nabla \vec{v}+\nabla \vec{v}^{\perp}\right|^{2}
$$

for all vector functions $\vec{v}$, where

$$
a_{i j}^{\alpha \beta}(X) D_{j} v^{\beta} D_{i} v^{\alpha}= \begin{cases}(\mu / 2)\left|\nabla \vec{v}+\nabla \vec{v}^{\perp}\right|^{2}+\lambda \operatorname{div}(\vec{v})^{2} & \text { for } X \in D, \\ (\tilde{\mu} / 2)\left|\nabla \vec{v}+\nabla \vec{v}^{\perp}\right|^{2}+\tilde{\lambda} \operatorname{div}(\vec{v})^{2} & \text { for } X \in \mathbf{R}^{n} \backslash D,\end{cases}
$$

and $\left|\nabla \vec{v}+\nabla \vec{v}^{\perp}\right|^{2}=\sum_{i, j=1}^{n}\left(D_{i} v^{j}+D_{j} v^{i}\right)^{2}$.

In this case the conormals associated to the operators in the interior and exterior of $D$ are given respectively by

$$
\begin{aligned}
& \frac{\partial \vec{u}^{+}}{\partial \nu}=\mu\left(\nabla \vec{u}^{+}+\nabla \vec{u}^{+\perp}\right) N+\lambda \operatorname{div}\left(\vec{u}^{+}\right) N \quad \text { and } \\
& \frac{\partial \vec{u}^{-}}{\partial \tilde{\nu}}=\tilde{\mu}\left(\nabla \vec{u}^{-}+\nabla \vec{u}^{-\perp}\right) N+\tilde{\lambda} \operatorname{div}\left(\vec{u}^{-}\right) N .
\end{aligned}
$$

We will now prove the following two theorems which are the analogues of Theorems 1 and 2 .

Theorem 3. Let $\vec{u}=\left(u^{1}, \ldots, u^{n}\right) \in\left[W^{1,2}(B)\right]^{n}$ be a weak solution to $S \vec{u}=0$ on $B$, where $S$ is as in (0.2), and $D$ be Lipschitz domain contained in $B_{1 / 2}$. Also, assume that (0.3) holds. Then, if $\mu \leq \tilde{\mu}$ and $\lambda \leq \tilde{\lambda}$ or $\mu \geq \tilde{\mu}$ and $\lambda \geq \tilde{\lambda}$, there is a constant $C$ depending on the Lipschitz character of $D, \mu, \tilde{\mu}, \lambda, \tilde{\lambda}$, and the smallest of the absolute value of the nonzero numbers $\mu-\tilde{\mu}$ and $\lambda-\dot{\lambda}$ such that $\left\|N\left(\nabla \vec{u}^{ \pm}\right)\right\|_{L^{2}(\partial D)} \leq C\|\vec{u}\|_{W^{1,2}(B)}$. 
Theorem 4. Let $\mu, \tilde{\mu}, \lambda, \tilde{\lambda}$, and $D$ be as in Theorem 3 , and $S$ and $\widetilde{S}$ denote respectively the single layer potentials on $\partial D$ associated to the elasticity operators with Lamé constants $\mu, \lambda$, and $\tilde{\mu}, \tilde{\lambda}$ respectively. Then the mapping

$$
\begin{gathered}
{\left[L^{2}(\partial D)\right]^{n} \times\left[L^{2}(\partial D)\right]^{n} \rightarrow\left[L_{1}^{2}(\partial D)\right]^{n} \times\left[L^{2}(\partial D)\right]^{n},} \\
(\vec{f}, \vec{g}) \rightarrow\left(S(\vec{f})-\widetilde{S}(\vec{g}), \frac{\partial}{\partial v} S(\vec{f})^{+}-\frac{\partial}{\partial \tilde{v}} \widetilde{S}(\vec{g})^{-}\right)
\end{gathered}
$$

is an invertible operator.

Proof of Theorem 3. Since the proof of this theorem is similar to the proof of Theorem 1 we will only sketch the main details. As in Theorem 1, we consider $\varphi \in C_{0}^{\infty}\left(\mathbf{R}^{n}\right)$ with $\varphi=1$ on $B_{3 / 4}$, and $\varphi=0$ outside $B_{7 / 8}$, and define $\vec{h}=\{\tilde{\mu} \Delta(\vec{u} \varphi)+(\tilde{\mu}+\tilde{\lambda}) \nabla(\operatorname{div}(\vec{u} \varphi))\} \chi_{\mathbf{R}^{n} \backslash D}$. As before we have $\|\vec{h}\|_{L^{2}\left(\mathbf{R}^{n}\right)} \leq$ $C\|\vec{u}\|_{W^{1,2(B)}}$.

We introduce the function $\vec{w}$ defined as

$$
\vec{w}=\vec{u} \varphi-\vec{\Gamma}(\vec{h})+\widetilde{S}(\vec{g}) \quad \text { for } X \in \mathbf{R}^{n} \backslash D, \quad \text { and } \quad \vec{w}=\vec{u}+S(\vec{f}) \text { for } X \in D \text {, }
$$

where $S$ and $\widetilde{S}$ are as in Theorem 4 , and $\vec{\Gamma}(\vec{h})$ denotes the Newtonian potential of $\vec{h}$ associated to the elasticity system outside $D$ (here $\vec{\Gamma}=\left(\Gamma^{i j}\right)$ as in (1.2) but with $\mu$ and $\lambda$ replaced by $\tilde{\mu}$ and $\tilde{\lambda}$ respectively). From Theorem 4 we can choose $\vec{f}$ and $\vec{g}$ in $\left[L^{2}(\partial D)\right]^{n}$ such that $S(\vec{f})-\widetilde{S}(\vec{g})=-\vec{\Gamma}(\vec{h})$ and

$$
\frac{\partial}{\partial v} S(\vec{f})^{+}-\frac{\partial}{\partial \tilde{v}} \widetilde{S}(\vec{g})^{-}=-\frac{\partial}{\partial \tilde{v}} \vec{\Gamma}(\vec{h}) \quad \text { on } \partial D .
$$

With this choice of $\vec{f}$ and $\vec{g}$ and as in Theorem 1 it is easy to check that $\vec{w} \in\left[W_{\mathrm{loc}}^{1,2}\left(\mathbf{R}^{n}\right)\right]^{n}$, and for all $\vec{\phi} \in\left[C_{0}^{\infty}\left(\mathbf{R}^{n}\right)\right]^{n}$

$$
\begin{aligned}
& \int_{D} \mu\left(\nabla \vec{w}+\nabla \vec{w}^{\perp}\right) \nabla \vec{\phi}+\lambda \operatorname{div}(\vec{w}) \operatorname{div}(\vec{\phi}) d X \\
& \quad+\int_{B \backslash D} \tilde{\mu}\left(\nabla \vec{w}+\nabla \vec{w}^{\perp}\right) \nabla \vec{\phi}+\tilde{\lambda} \operatorname{div}(\vec{w}) \operatorname{div}(\vec{\phi}) d X=0 .
\end{aligned}
$$

Now we will show that $\vec{w}=0$. Let $\eta \in C_{0}^{\infty}\left(B_{2 r}\right)$ with $\eta=1$ on $B_{r}$. From Plancherel equality, the Legendre-Hadamard condition, and (3.1) we have

$$
\begin{aligned}
& \int|\nabla(\vec{w} \eta)|^{2} d X \leq C \int_{D} \frac{\mu}{2}\left|\nabla(\vec{w} \eta)+\nabla\left(\vec{w}^{\perp} \eta\right)\right|^{2}+\lambda \operatorname{div}((\vec{w} \eta))^{2} d X \\
& \leq C \int\left[\frac{\mu}{2} \chi_{D}+\frac{\tilde{\mu}}{2} \chi_{\mathbf{R}^{n} \backslash D}\right]\left|\nabla(\vec{w} \eta)+\nabla\left(\vec{w}^{\perp} \eta\right)\right|^{2} \\
&+\left[\lambda \chi_{D}+\tilde{\lambda} \chi_{\mathbf{R}^{n} \backslash D}\right] \operatorname{div}((\vec{w} \eta))^{2} d X .
\end{aligned}
$$

The above estimates also hold when $\vec{w}$ is replaced by $\vec{w}-\vec{w}_{r}$, where $\vec{w}_{r}$ denotes the average of $\vec{w}$ on $B_{2 r} \backslash B_{r}$. These and standard arguments [G, Chapter 3, Proposition 2.1] imply

$$
\int_{B_{r}}|\nabla \vec{w}|^{2} d X \leq \theta \int_{B_{2 r}}|\nabla \vec{w}|^{2} d X \quad \text { for all } r>0,
$$

where $\theta$ is a number in the interval $(0,1)$ depending on $\mu, \tilde{\mu}, \lambda$, and $\tilde{\lambda}$. Since $\nabla w(X)=O\left(|X|^{1-n}\right)$, the above inequality implies after letting $r$ tend to infinity 
that $\nabla \vec{w}$ is identically zero on $\mathbf{R}^{n}$. The behavior of $\vec{w}$ at infinity shows that $\vec{w}$ is identically zero on $\mathbf{R}^{n}$. Thus, we obtain as in Theorem 1 a representation formula for $\vec{u}$ in a neighborhood of $D$, which combined with Theorem $4,(1.5)$, and the estimate $\|\nabla \vec{\Gamma}(\vec{h})\|_{L^{\infty}\left(B_{3 / 4}\right)} \leq C\|\vec{u}\|_{W^{1,2}(B)}$ proves Theorem 3.

Proof of Theorem 4. Let $S$ and $\widetilde{S}$ be as in Theorem 4. An argument similar to the one we gave in Theorem 3 to show that the function $\vec{w}$ is identically zero shows that when $\vec{f}$ and $\vec{g} \in\left[L^{2}(2 D)\right]^{n}$ satisfy

$$
S(\vec{f})-\tilde{S}(\vec{g})=0, \quad \frac{\partial}{\partial v} S(\vec{f})^{+}-\frac{\partial}{\partial \tilde{v}} \tilde{S}(\vec{g})^{-}=0 \quad \text { on } \partial D
$$

then $S(\vec{f})$ and $\tilde{S}(\vec{g})$ are identically zero in the interior and exterior of $D$ respectively. Since

$$
\int_{\mathbf{R}^{n} \backslash D} \frac{\mu}{2}\left|\nabla S(\vec{f})+\nabla S(\vec{f})^{\perp}\right|^{2}+\lambda \operatorname{div}(S(\vec{f}))^{2}=-\int_{\partial D} S(\vec{f}) \frac{\partial}{\partial v} S(\vec{f})^{-} d \sigma
$$

and

$$
\int_{D} \frac{\tilde{\mu}}{2}\left|\nabla \tilde{S}(\vec{g})+\nabla \tilde{S}(\vec{g})^{\perp}\right|^{2}+\tilde{\lambda} \operatorname{div}(\tilde{S}(\vec{g}))^{2}=\int_{\partial D} \tilde{S}(\vec{g}) \frac{\partial}{\partial \tilde{v}} \tilde{S}(\vec{g})^{+} d \sigma
$$

we have $S(\vec{f})=\tilde{S}(\vec{g})=0$ on $\mathbf{R}^{n}$, and as in Theorem 2 we get from the jump relations of the tractions $(1.6), \vec{f}=\vec{g}=0 d$ on $\partial D$. Therefore, the operator is one-to-one.

As in Theorem 1 to show that the range is $\left[L^{2}(\partial D)\right]^{n}$ it suffices to obtain an estimate of the form

$$
\begin{aligned}
&\|\vec{f}\|_{L^{2}(\partial D)}+\|\vec{g}\|_{L^{2}(\partial D)} \\
& \leq C\left\{\|S(\vec{f})-\tilde{S}(\vec{g})\|_{L_{1}^{2}(\partial D)}+\left\|\frac{\partial}{\partial v} S(\vec{f})^{+}-\frac{\partial}{\partial \tilde{v}} \tilde{S}(\vec{g})^{-}\right\|_{L^{2}(\partial D)}\right. \\
&\left.+\left|L_{+}(\vec{f})\right|+\left|L_{-}(\vec{g})\right|\right\},
\end{aligned}
$$

where $L_{+}$and $L_{-}$denote bounded linear operators on $\left[L^{2}(\partial D)\right]^{n}$ whose norm only depends on the Lipschitz character of $D$.

Setting $\vec{u}^{+}=S(\vec{f}), \vec{u}^{-}=\widetilde{S}(\vec{g}), \vec{h}_{1}=S(\vec{f})-\widetilde{S}(\vec{g})$, and $\vec{h}_{2}=\partial S(\vec{f})^{+} / \partial v-$ $\partial \widetilde{S}(\vec{g})^{-} / \partial \tilde{v}$ we will show that

$$
\begin{aligned}
& \left\|\nabla \vec{u}^{+}\right\|_{L^{2}(\partial D)}+\left\|\nabla \vec{u}^{-}\right\|_{L^{2}(\partial D)} \\
& \leq C\left\{\left\|\vec{h}_{1}\right\|_{L_{1}^{2}(\partial D)}+\left\|\vec{h}_{2}\right\|_{L^{2}(\partial D)}+\left\|\nabla \vec{u}^{+}+\nabla \vec{u}^{+\perp}\right\|_{L^{2}(D)}\right. \\
& \left.\quad+\left\|\nabla \vec{u}^{-}+\nabla \vec{u}^{-\perp}\right\|_{L^{2}(B \backslash D)}+\left|l_{+}\left(\vec{u}^{+}\right)\right|+\left|l_{-}\left(\vec{u}^{-}\right)\right|\right\},
\end{aligned}
$$

where $l_{+}$and $l_{-}$are respectively linear forms on $W^{1,2}(B)$ and $W^{1,2}(B \backslash D)$, whose norms depend respectively on the measure in $\mathbf{R}^{n}$ of $D$ and $B \backslash D$, and with $C$ as in Theorem 4 . On the other hand, from [D, K, V, 2] there is a constant $C$ with the same dependence, so that

$$
\begin{aligned}
& \|\vec{f}\|_{L^{2}(\partial D)} \leq C\left\{\left\|\nabla \vec{u}^{+}\right\|_{L^{2}(\partial D)}+\left|L_{+}(\vec{f})\right|\right\}, \\
& \|\vec{g}\|_{L^{2}(\partial D)} \leq C\left\{\left\|\nabla \vec{u}^{-}\right\|_{L^{2}(\partial D)}+\left|L_{-}(\vec{g})\right|\right\} .
\end{aligned}
$$


Once we have proved (3.3), inequality (3.2) will follow from (3.3), (3.4), the identities

$$
\begin{aligned}
\int_{D} \frac{\mu}{2}\left|\nabla \vec{u}^{+}+\nabla \vec{u}^{+\perp}\right|^{2}+\lambda \operatorname{div}\left(\vec{u}^{+}\right)^{2} & =\int_{\partial D} \frac{\vec{u}^{+} \partial}{\partial v} \vec{u}^{+} d \sigma \\
\int_{\mathbf{R}^{n} \backslash D} \frac{\tilde{\mu}}{2}\left|\nabla \vec{u}^{-}+\nabla \vec{u}^{-\perp}\right|^{2}+\tilde{\lambda} \operatorname{div}\left(\vec{u}^{-}\right)^{2} & =-\int_{\partial D} \vec{u}^{-} \frac{\partial}{\partial \tilde{v}} \vec{u}^{-} d \sigma,
\end{aligned}
$$

(3.1), and Theorem 0 .

To prove (3.3) the following formulas will be of use to us. Let $\left\{N, T_{1}, \ldots\right.$, $\left.T_{n-1}\right\}$ be the orthonormal basis defined in $\S 1$ and associated to almost every point $P \in \partial D$, where $N$ is the exterior unit normal at $P$, and $T_{1}, \ldots, T_{n-1}$ are $n-1$ tangential vectors at $P$ on $\partial D$. Then, for any vector valued function $\vec{v}$

$$
\left|\nabla \vec{v}+\nabla \vec{v}^{\perp}\right|^{2}=\left\langle\left(\nabla \vec{v}+\nabla \vec{v}^{\perp}\right) N, N\right\rangle^{2}+2 \sum_{i=1}^{n-1}\left\langle\left(\nabla \vec{v}+\nabla \vec{v}^{\perp}\right) N, T_{i}\right\rangle^{2}
$$

$$
+\sum_{i, j=1}^{n-1}\left\langle\left(\nabla \vec{v}+\nabla \vec{v}^{\perp}\right) T_{i}, T_{j}\right\rangle^{2}
$$

$$
\begin{gathered}
\operatorname{div}(\vec{v})=\frac{1}{2}\left\{\left\langle\left(\nabla \vec{v}+\nabla \vec{v}^{\perp}\right) N, N\right\rangle+\sum_{i=1}^{n-1}\left\langle\left(\nabla \vec{v}+\nabla \vec{v}^{\perp}\right) T_{i}, T_{i}\right\rangle\right\}, \\
\left\langle\frac{\partial}{\partial v} \vec{v}, N\right\rangle=\frac{2 \mu+\lambda}{2}\left\langle\left(\nabla \vec{v}+\nabla \vec{v}^{\perp}\right) N, N\right\rangle+\frac{\lambda}{2} \sum_{i=1}^{n-1}\left\langle\left(\nabla \vec{v}+\nabla \vec{v}^{\perp}\right) T_{i}, T_{i}\right\rangle, \\
\left\langle\frac{\partial}{\partial v} \vec{v}, T_{i}\right\rangle=\mu\left\langle\left(\nabla \vec{v}+\nabla \vec{v}^{\perp}\right) N, T_{i}\right\rangle \text { for all } i=1, \ldots, n-1 .
\end{gathered}
$$

These identities derive from the orthogonality of the base $\left\{N, T_{1}, \ldots, T_{n-1}\right\}$. From the above identities we have

$$
\begin{aligned}
\lambda \operatorname{div}(\vec{v})^{2} & +\frac{\mu}{2}\left|\nabla \vec{v}+\nabla \vec{v}^{\perp}\right|^{2} \\
= & \frac{1}{2 \mu+\lambda}\left\langle\frac{\partial}{\partial v} \vec{v}, N\right\rangle^{2}+\frac{1}{\mu} \sum_{i=1}^{n-1}\left\langle\frac{\partial}{\partial v} \vec{v}, T_{i}\right\rangle^{2} \\
& +\frac{\mu \lambda}{2(2 \mu+\lambda)}\left[\sum_{i=1}^{n-1}\left\langle\left(\nabla \vec{v}+\nabla \vec{v}^{\perp}\right) T_{i}, T_{i}\right\rangle\right]^{2} \\
& +\frac{\mu}{2} \sum_{i, j=1}^{n-1}\left\langle\left(\nabla \vec{v}+\nabla \vec{v}^{\perp}\right) T_{i}, T_{j}\right\rangle^{2} .
\end{aligned}
$$

As in Theorem 2, we will consider a vector field $\vec{\beta} \in C_{0}^{\infty}\left(B_{3 / 4}\right)$ so that $\langle\vec{\beta}, N\rangle \geq C$, with $C$ depending only on the Lipschitz character of $D[\mathrm{~V}]$, and the following Rellich-Necas [N] identities:

$$
\begin{aligned}
\operatorname{div} & \left(\vec{\beta}\left[\lambda \operatorname{div}\left(\vec{u}^{+}\right)^{2}+\frac{\mu}{2}\left|\nabla \vec{u}^{+}+\nabla \vec{u}^{+\perp}\right|^{2}\right]\right) \\
& =2 \operatorname{div}\left(\vec{\beta}_{i} D_{i} \vec{u}^{+} \cdot\left\{\lambda \operatorname{div}\left(\vec{u}^{+}\right) I+\frac{\mu}{2}\left(\nabla \vec{u}^{+}+\nabla \vec{u}^{+\perp}\right)\right\}\right)+O\left(\left|\nabla \vec{u}^{+}\right|^{2}\right) \text { on } D,
\end{aligned}
$$




$$
\begin{array}{r}
\operatorname{div}\left(\vec{\beta}\left[\tilde{\lambda} \operatorname{div}\left(\vec{u}^{-}\right)^{2}+\frac{\tilde{\mu}}{2}\left|\nabla \vec{u}^{-}+\nabla \vec{u}^{-\perp}\right|^{2}\right]\right) \\
=2 \operatorname{div}\left(\vec{\beta}_{i} D_{i} \vec{u}^{-} \cdot\left\{\tilde{\lambda} \operatorname{div}\left(\vec{u}^{-}\right) I+\frac{\tilde{\mu}}{2}\left(\nabla \vec{u}^{-}+\nabla \vec{u}^{-\perp}\right)\right\}\right)+O\left(\left|\nabla \vec{u}^{-}\right|^{2}\right) \\
\quad \text { on } \mathbf{R}^{n} \backslash D,
\end{array}
$$

where $I$ denotes the identity matrix and - the multiplication of matrices. Interating on $D$ the first identity above we get

$$
\begin{gathered}
\int_{\partial D}\langle\vec{\beta}, N\rangle\left\{\lambda \operatorname{div}\left(\vec{u}^{+}\right)^{2}+\frac{\mu}{2}\left|\nabla \vec{u}^{+}+\nabla \vec{u}^{+\perp}\right|^{2}\right\} d \sigma \\
=2 \int_{\partial D} \vec{\beta}_{i} D_{i} \vec{u}^{+} \frac{\partial}{\partial v} \vec{u}^{+} d \sigma+O\left(\left\|\nabla \vec{u}^{+}\right\|_{L^{2}(D)}^{2}\right) .
\end{gathered}
$$

Observing that

$$
\begin{aligned}
\vec{\beta}_{i} D_{i} \vec{u}^{+} \frac{\partial}{\partial v} \vec{u}^{+}= & \langle\vec{\beta}, N\rangle\left[\frac{1}{2}\left\langle\frac{\partial}{\partial v} \vec{u}^{+}, N\right\rangle\left\langle\left(\nabla \vec{u}^{+}+\nabla \vec{u}^{+\perp}\right) N, N\right\rangle\right. \\
& \left.+\left\langle\nabla \vec{u}^{+} T_{i}, N\right\rangle\left\langle\frac{\partial}{\partial v} \vec{u}^{+}, T_{i}\right\rangle\right] \\
& +\left\langle\vec{\beta}, T_{i}\right\rangle\left\langle\nabla \vec{u}^{+} \frac{\partial}{\partial v} \vec{u}^{+}, T_{i}\right\rangle,
\end{aligned}
$$

we get the following formula after substituting (3.9) and (3.11) in (3.10):

$$
\begin{gathered}
\int_{\partial D}\langle\vec{\beta}, N\rangle\left\{\frac{1}{2 \mu+\lambda}\left\langle\frac{\partial}{\partial v} \vec{u}^{+}, N\right\rangle^{2}+\frac{1}{\mu} \sum_{i=1}^{n-1}\left\langle\frac{\partial}{\partial v} \vec{u}^{+}, T_{i}\right\rangle^{2}\right. \\
+\frac{\mu \lambda}{2(2 \mu+\lambda)}\left[\sum_{i=1}^{n-1}\left\langle\left(\nabla \vec{u}^{+}+\nabla \vec{u}^{+\perp}\right) T_{i}, T_{i}\right\rangle\right]^{2} \\
+\frac{\mu}{2} \sum_{i, j=1}^{n-1}\left\langle\left(\nabla \vec{u}^{+}+\nabla \vec{u}^{+\perp}\right) T_{i}, T_{j}\right\rangle^{2} \\
\quad-\left\langle\left(\nabla \vec{u}^{+}+\nabla \vec{u}^{+\perp}\right) N, N\right\rangle\left\langle\frac{\partial}{\partial v} \vec{u}^{+}, N\right\rangle \\
=2 \int_{\partial D} \sum_{i=1}^{n-1}\left\langle\vec{\beta}, T_{i}\right\rangle\left\langle\nabla \vec{u}^{+} \frac{\partial}{\partial v} \vec{u}^{+}, T_{i}\right\rangle d \sigma+O\left(\left\|\nabla \vec{u}^{+}\right\|_{L^{2}(D)}^{2}\right) .
\end{gathered}
$$

From the identities

$$
\begin{aligned}
\frac{1}{2 \mu+\lambda} & \left\langle\frac{\partial}{\partial v} \vec{u}^{+}, N\right\rangle^{2}-\left\langle\left(\nabla \vec{u}^{+}+\nabla \vec{u}^{+\perp}\right) N, N\right\rangle\left\langle\frac{\partial}{\partial v} \vec{u}^{+}, N\right\rangle \\
= & -\frac{1}{2 \mu+\lambda}\left\langle\frac{\partial}{\partial v} \vec{u}^{+}, N\right\rangle^{2} \\
& +\frac{\lambda}{2 \mu+\lambda}\left\langle\frac{\partial}{\partial v} \vec{u}^{+}, N\right\rangle \sum_{i=1}^{n-1}\left\langle\left(\nabla \vec{u}^{+}+\nabla \vec{u}^{+\perp}\right) T_{i}, T_{i}\right\rangle,
\end{aligned}
$$




$$
\begin{array}{r}
\frac{1}{\mu} \sum_{i=1}^{n-1}\left\langle\frac{\partial}{\partial v} \vec{u}^{+}, T_{i}\right\rangle^{2}-2 \sum_{i=1}^{n-1}\left\langle\nabla \vec{u}^{+} T_{i}, N\right\rangle\left\langle\frac{\partial}{\partial v} \vec{u}^{+}, T_{i}\right\rangle \\
=\sum_{i=1}^{n-1}\left\langle\frac{\partial}{\partial v} \vec{u}^{+}, T_{i}\right\rangle\left(\left\langle\nabla \vec{u}^{+} N, T_{i}\right\rangle-\left\langle\nabla \vec{u}^{+\perp} N, T_{i}\right\rangle\right),
\end{array}
$$

and (3.12) we obtain

$$
\begin{aligned}
& \int_{\partial D}\langle\vec{\beta}, N\rangle\{- \frac{1}{2 \mu+\lambda}\left\langle\frac{\partial}{\partial v} \vec{u}^{+}, N\right\rangle^{2} \\
&+\frac{\lambda}{2 \mu+\lambda}\left\langle\frac{\partial}{\partial v} \vec{u}^{+}, N\right\rangle\left[\sum_{i=1}^{n-1}\left\langle\left(\nabla \vec{u}^{+}+\nabla \vec{u}^{+\perp}\right) T_{i}, T_{i}\right\rangle\right] \\
&+\frac{\mu \lambda}{2(2 \mu+\lambda)}\left[\sum_{i=1}^{n-1}\left\langle\left(\nabla \vec{u}^{+}+\nabla \vec{u}^{+\perp}\right) T_{i}, T_{i}\right\rangle\right]^{2} \\
&+\frac{\mu}{2} \sum_{i, j=1}^{n-1}\left\langle\left(\nabla \vec{u}^{+}+\nabla \vec{u}^{+\perp}\right) T_{i}, T_{j}\right\rangle^{2} \\
&\left.+\sum_{i=1}^{n-1}\left\langle\frac{\partial}{\partial v} \vec{u}^{+}, T_{i}\right\rangle\left(\left\langle\nabla \vec{u}^{+} N, T_{i}\right\rangle-\left\langle\nabla \vec{u}^{+\perp} N, T_{i}\right\rangle\right)\right\} d \sigma \\
&=2 \int_{\partial D} \sum_{i=1}^{n-1}\left\langle\vec{\beta}, T_{i}\right\rangle\left\langle\nabla \vec{u}^{+} \frac{\partial}{\partial v} \vec{u}^{+}, T_{i}\right\rangle d \sigma+O\left(\left\|\nabla \vec{u}^{+}\right\|_{L^{2}(D)}^{2}\right) .
\end{aligned}
$$

The same argument leads to an identity similar to (3.15) but with $\vec{u}^{+}, \mu, \lambda$, and $\partial / \partial v$ replaced respectively by $\vec{u}^{-}, \tilde{\mu}, \tilde{\lambda}$, and $\partial / \partial \tilde{v}$. Since $\vec{h}_{1}=\vec{u}^{+}-\vec{u}^{-}$ and $\vec{h}_{2}=\partial \vec{u}^{+} \partial v-\partial \vec{u}^{+} \vec{u}^{-} \partial \tilde{v}$ on $\partial D$, and from (3.7) and (3.8) it is easy to rewrite this second identity by replacing $\vec{u}^{-}$by $\vec{u}^{+}$to obtain the formula

$$
\begin{aligned}
\int_{\partial D}\langle\vec{\beta}, N\rangle\{ & -\frac{1}{2 \tilde{\mu}+\tilde{\lambda}}\left\langle\frac{\partial}{\partial v} \vec{u}^{+}, N\right\rangle^{2} \\
& +\frac{\tilde{\lambda}}{2 \tilde{\mu}+\tilde{\lambda}}\left\langle\frac{\partial}{\partial v} \vec{u}^{+}, N\right\rangle\left[\sum_{i=1}^{n-1}\left\langle\left(\nabla \vec{u}^{+}+\nabla \vec{u}^{+\perp}\right) T_{i}, T_{i}\right\rangle\right] \\
& +\frac{\tilde{\mu} \tilde{\lambda}}{2(2 \tilde{\mu}+\tilde{\lambda})}\left[\sum_{i=1}^{n-1}\left\langle\left(\nabla \vec{u}^{+}+\nabla \vec{u}^{+\perp}\right) T_{i}, T_{i}\right\rangle\right]^{2} \\
& +\frac{\tilde{\mu}}{2} \sum_{i, j=1}^{n-1}\left\langle\left(\nabla \vec{u}^{+}+\nabla \vec{u}^{+\perp}\right) T_{i}, T_{j}\right\rangle^{2} \\
& \left.+\sum_{i=1}^{n-1}\left\langle\frac{\partial}{\partial v} \vec{u}^{+}, T_{i}\right\rangle\left(\frac{2 \tilde{\mu}-\mu}{\tilde{\mu}}\left\langle\nabla \vec{u}^{+} N, T_{i}\right\rangle-\frac{\mu}{\tilde{\mu}}\left\langle\nabla \vec{u}^{+\perp} N, T_{i}\right\rangle\right)\right\} d \sigma
\end{aligned}
$$




$$
\begin{aligned}
= & 2 \int_{\partial D} \sum_{i=1}^{n-1}\left\langle\vec{\beta}, T_{i}\right\rangle\left\langle\nabla \vec{u}^{+} \frac{\partial}{\partial v} \vec{u}^{+}, T_{i}\right\rangle d \sigma \\
& +O\left(\left\|\nabla \vec{u}^{-}\right\|_{L^{2}(B \backslash D)}^{2}+\left\|\vec{h}_{1}\right\|_{L_{1}^{2}(\partial D)}\right. \\
& +\left\|\vec{h}_{2}\right\|_{L^{2}(\partial D)} \\
& \left.+\left\|\nabla \vec{u}^{+}\right\|_{L^{2}(\partial D)}\left\{\left\|\vec{h}_{1}\right\|_{L_{1}^{2}(\partial D)}+\left\|\vec{h}_{2}\right\|_{L^{2}(\partial D)}\right\}\right) .
\end{aligned}
$$

Subtracting (3.16) from (3.15) and using the identities (3.7) and (3.8) we get

$$
\begin{aligned}
\int_{\partial D}\langle\vec{\beta}, N\rangle\{ & A\left\langle\left(\nabla \vec{u}^{+}+\nabla \vec{u}^{+\perp}\right) N, N\right\rangle^{2}+B\left[\sum_{i=1}^{n-1}\left\langle\left(\nabla \vec{u}^{+}+\nabla \vec{u}^{+\perp}\right) T_{i}, T_{i}\right\rangle\right]^{2} \\
& +C\left\langle\left(\nabla \vec{u}^{+}+\nabla \vec{u}^{+\perp}\right) N, N\right\rangle\left[\sum_{i=1}^{n-1}\left\langle\left(\nabla \vec{u}^{+}+\nabla \vec{u}^{+\perp}\right) T_{i}, T_{i}\right\rangle\right] \\
& +D \sum_{i=1}^{n-1}\left\langle\left(\nabla \vec{u}^{+}+\nabla \vec{u}^{+\perp}\right) N, T_{i}\right\rangle^{2} \\
& \left.+E \sum_{i, j=1}^{n-1}\left\langle\left(\nabla \vec{u}^{+}+\nabla \vec{u}^{+\perp}\right) T_{i}, T_{j}\right\rangle^{2}\right\} d \sigma \\
=O\left(\left\|\nabla \vec{u}^{+}\right\|_{L^{2}(D)}^{2}+\left\|\nabla \vec{u}^{-}\right\|_{L^{2}(B \backslash D)}^{2}+\left\|\nabla \vec{u}^{+}\right\|_{L^{2}(\partial D)}\left\{\left\|\vec{h}_{1}\right\|_{L_{1}^{2}(\partial D)}+\left\|\vec{h}_{2}\right\|_{L^{2}(\partial D)}\right\}\right. & \left.+\left\|\vec{h}_{1}\right\|_{L_{1}^{2}(\partial D)}+\left\|\vec{h}_{2}\right\|_{L^{2}(\partial D)}\right),
\end{aligned}
$$

where

$$
\begin{aligned}
& A=\frac{(2 \mu+\lambda)(2(\mu-\tilde{\mu})+(\lambda-\tilde{\lambda}))}{2 \tilde{\mu}+\tilde{\lambda}}, \quad B=\frac{\left(4 \mu \tilde{\mu}+2(\mu+\tilde{\mu}) \lambda+\lambda^{2}\right)(\lambda-\tilde{\lambda})}{(2 \mu+\lambda)(2 \tilde{\mu}+\tilde{\lambda})}, \\
& C=\frac{2(2 \mu+\lambda)(\lambda-\tilde{\lambda})}{2 \tilde{\mu}+\tilde{\lambda}}, \quad D=\frac{4 \mu(\mu-\tilde{\mu})}{\tilde{\mu}}, \quad \text { and } \quad E=2(\mu-\tilde{\mu}) .
\end{aligned}
$$

A short calculation shows that the determinant of the $2 \times 2$ matrix

$$
\left[\begin{array}{cc}
A & \frac{1}{2} C \\
\frac{1}{2} C & B
\end{array}\right]
$$

equals $(\lambda-\tilde{\lambda})(\mu-\tilde{\mu})(2 \mu+\lambda) /(2 \tilde{\mu}+\tilde{\lambda})$. Therefore, when $\mu>\tilde{\mu}$ and $\lambda>\tilde{\lambda}$ the above matrix is definite positive, and from (3.17) and (3.5) we get

$$
\begin{aligned}
& \left\|\nabla \vec{u}^{+}+\nabla \vec{u}^{+\perp}\right\|_{L^{2}(\partial D)}^{2} \\
& \leq C\left\{\left\|\vec{h}_{1}\right\|_{L_{1}^{2}(\partial D)}^{2}+\left\|\vec{h}_{2}\right\|_{L^{2}(\partial D)}^{2}+\left\|\nabla \vec{u}^{+}\right\|_{L^{2}(\partial D)}\left[\left\|\vec{h}_{1}\right\|_{L_{1}^{2}(\partial D)}+\left\|\vec{h}_{2}\right\|_{L^{2}(\partial D)}\right]\right. \\
& \left.+\left\|\nabla \vec{u}^{+}\right\|_{L^{2}(D)}^{2}+\left\|\nabla \vec{u}^{-}\right\|_{L^{2}(B \backslash D)}^{2}\right\} .
\end{aligned}
$$

The same reasoning shows that the above estimate holds when $\mu<\tilde{\mu}$ and $\lambda<\tilde{\lambda}$. At this point we recall the following form of Korn's boundary inequality (see [D, K, V, 2]). 
Theorem 5. Let $\vec{u}$ satisfy $\mu \Delta \vec{u}+(\lambda+\mu) \nabla(\operatorname{div} \vec{u})=0$ in the interior of a bounded Lipschitz domain $D$ contained in $B_{1 / 2}$ (respectively on $B \backslash D$ ), with Lamé constants $\mu$ and $\lambda, \mu>0$, and $\lambda>-2 \mu / n$. Then there is a constant $C$ depending on the Lipschitz character of $D, \mu$, and $\lambda$ so that

$$
\|\nabla \vec{u}\|_{L^{2}(\partial D)} \leq C\left\{\left\|\nabla \vec{u}+\nabla \vec{u}^{\perp}\right\|_{L^{2}(\partial D)}+|l(\vec{u})|\right\},
$$

where $l$ is a linear form on $W_{1}^{2}(D) \quad\left(W_{1}^{2}(B \backslash D)\right)$ whose norm only depends on the measure in $\mathbf{R}^{n}$ of $D(B \backslash D)$.

On the other hand, from the classical Korn's [F] inequality we have

$$
\begin{aligned}
\left\|\nabla \vec{u}^{+}\right\|_{L^{2}(D)} & \leq C\left\{\left\|\nabla \vec{u}^{+}+\nabla \vec{u}^{+\perp}\right\|_{L^{2}(D)}+\left|l_{+}\left(\vec{u}^{+}\right)\right|\right\}, \\
\left\|\nabla \vec{u}^{-}\right\|_{L^{2}(B \backslash D)} & \leq C\left\{\left\|\nabla \vec{u}^{-}+\nabla \vec{u}^{-\perp}\right\|_{L^{2}(B \backslash D)}+\left|l_{-}\left(\vec{u}^{-}\right)\right|\right\},
\end{aligned}
$$

where $l_{+}$and $l_{-}$are as in (3.3). From these inequalities, Theorem 5 , the transmission conditions, and (3.18) we obtain (3.3) when $\mu<\tilde{\mu}$ and $\lambda<\tilde{\lambda}$ or $\mu>\tilde{\mu}$ and $\lambda>\tilde{\lambda}$.

Also observe that when $\lambda=\tilde{\lambda}$ the integrand in the left-hand side of (3.17) can be rewritten as

$$
\begin{aligned}
(\mu-\tilde{\mu})\left[\langle \vec { \beta } , N \rangle \left\{\frac{2(2 \mu+\lambda)}{2 \tilde{\mu}+\lambda}\left\langle\left(\nabla \vec{u}^{+}+\nabla \vec{u}^{+\perp}\right) N, N\right\rangle^{2}\right.\right. \\
+4 \frac{\mu}{\tilde{\mu}} \sum_{i=1}^{n-1}\left\langle\left(\nabla \vec{u}^{+}+\nabla \vec{u}^{+\perp}\right) N, T_{i}\right\rangle^{2} \\
\left.\left.+2 \sum_{i, j=1}^{n-1}\left\langle\left(\nabla \vec{u}^{+}+\nabla \vec{u}^{+\perp}\right) T_{i}, T_{j}\right\rangle^{2}\right\}\right]
\end{aligned}
$$

which as above gives (3.18).

When $\mu=\tilde{\mu}$ and $\lambda \neq \tilde{\lambda}$, and using (3.6), the integrand in (3.17) can be rewritten as

$$
\frac{2(2 \mu+\lambda)(\lambda-\tilde{\lambda})}{2 \tilde{\mu}+\tilde{\lambda}}\langle\vec{\beta}, N\rangle \operatorname{div}\left(\vec{u}^{+}\right)^{2},
$$

to obtain the estimate

$$
\begin{aligned}
\left\|\operatorname{div}\left(\vec{u}^{+}\right)\right\|_{L^{2}(\partial D)}^{2} \leq C\left\{\left\|\vec{h}_{1}\right\|_{L_{1}^{2}(\partial D)}^{2}+\left\|\vec{h}_{2}\right\|_{L^{2}(\partial D)}^{2}\right. & \\
+\left\|\nabla \vec{u}^{+}\right\|_{L^{2}(\partial D)} & {\left[\left\|\vec{h}_{1}\right\|_{L_{1}^{2}(\partial D)}+\left\|\vec{h}_{2}\right\|_{L^{2}(\partial D)}\right] } \\
& \left.+\left\|\nabla \vec{u}^{+}\right\|_{L^{2}(D)}^{2}+\left\|\nabla \vec{u}^{-}\right\|_{L^{2}(B \backslash D}^{2}\right\} .
\end{aligned}
$$

Observe that in this case the same argument gives

$$
\begin{aligned}
\left\|\operatorname{div}\left(\vec{u}^{-}\right)\right\|_{L^{2}(\partial D)}^{2} \leq C\left\{\left\|\vec{h}_{1}\right\|_{L_{1}^{2}(\partial D)}^{2}+\left\|\vec{h}_{2}\right\|_{L^{2}(\partial D)}^{2}\right. & \\
+\left\|\nabla \vec{u}^{-}\right\|_{L^{2}(\partial D)} & {\left[\left\|\vec{h}_{1}\right\|_{L_{1}^{2}(\partial D)}+\left\|\vec{h}_{2}\right\|_{L^{2}(\partial D)}\right] } \\
& \left.+\left\|\nabla \vec{u}^{+}\right\|_{L^{2}(D)}^{2}+\left\|\nabla \vec{u}^{-}\right\|_{L^{2}(B \backslash D)}^{2}\right\} .
\end{aligned}
$$


At this point we will proceed as in the last case of Theorem 2. The weak formulation of the equation satisfied by $\vec{u}$ on $\mathbf{R}^{n}$ can be rewritten as

$$
\begin{aligned}
\int_{\mathbf{R}^{n}} \mu & \left(\nabla \vec{u}+\nabla \vec{u}^{\perp}\right) \nabla \vec{\phi} d X \\
= & -\int_{D} \lambda \operatorname{div}(\vec{u})\left(\operatorname{div}(\vec{\phi}) d X-\int_{\mathbf{R}^{n} \backslash D} \tilde{\lambda} \operatorname{div}(\vec{u}) \operatorname{div}(\vec{\phi}) d X\right. \\
= & \int_{\mathbf{R}^{n}}\left[\lambda \nabla \operatorname{div}\left(\vec{u}^{+}\right) \chi_{D}+\tilde{\lambda} \nabla \operatorname{div}\left(\vec{u}^{-}\right) \chi_{\mathbf{R}^{n} \backslash D}\right] \vec{\phi} d X \\
& +\int_{\partial D}\left\{\vec{h}_{2}+\left[\tilde{\lambda} \operatorname{div}\left(\vec{u}^{-}\right)-\lambda \operatorname{div}\left(\vec{u}^{+}\right)\right] N\right\} \vec{\phi} d \sigma
\end{aligned}
$$

for all test functions $\vec{\phi} \in\left[C_{0}^{\infty}\left(\mathbf{R}^{n}\right)\right]^{n}$, and $\vec{u}^{+}-\vec{u}^{-}=\vec{h}_{1}$ on $\partial D$.

If we let $\vec{\Gamma}(X)$ and $\widetilde{\widetilde{S}}$ denote respectively the matrix fundamental solution, and single layer potential of the system of elastostatics with Lamé constants $\mu>0$ and $\lambda=0$, and $\vec{w}$ be the solution to $\mu \Delta \vec{w}+\mu \nabla \operatorname{div} \vec{w}=0$ on $D$, $\vec{w}=\vec{h}_{1}$ on $\partial D$, and define the function

$$
\begin{aligned}
\vec{v}= & \tilde{\widetilde{S}}\left(\vec{h}_{2}+\left[\tilde{\lambda} \operatorname{div}\left(\vec{u}^{-}\right)-\lambda \operatorname{div}\left(\vec{u}^{+}\right)\right] N+\mu\left(\nabla \vec{w}+\nabla \vec{w}^{\perp}\right) N\right) \\
& -\vec{\Gamma}\left(\lambda \nabla \operatorname{div}\left(\vec{u}^{+}\right) \chi_{D}+\tilde{\lambda} \nabla \operatorname{div}\left(\vec{u}^{-}\right) \chi_{\mathbf{R}^{n} \backslash D}\right) \quad \text { on } \mathbf{R}^{n} \backslash D,
\end{aligned}
$$

and

$$
\begin{aligned}
\vec{v}= & \vec{w}+\tilde{\widetilde{S}}\left(\vec{h}_{2}+\left[\tilde{\lambda} \operatorname{div}\left(\vec{u}^{-}\right)-\lambda \operatorname{div}\left(\vec{u}^{+}\right)\right] N+\mu\left(\nabla \vec{w}+\nabla \vec{w}^{\perp}\right) N\right) \\
& \left.-\vec{\Gamma}\left(\lambda \nabla \operatorname{div}\left(\vec{u}^{+}\right) \chi_{D}+\tilde{\lambda} \nabla \operatorname{div}(\vec{u})^{-}\right) \chi_{\mathbf{R}^{n} \backslash D}\right) \quad \text { on } D,
\end{aligned}
$$

it turns out from $(3.21)$ and (1.6) that $\vec{v}-\vec{u}$ satisfies $\mu \Delta(\vec{v}-\vec{u})+\mu \nabla(\operatorname{div}(\vec{v}-\vec{u}))=$ 0 on $\mathbf{R}^{n}$. From Lemma 1 and its obvious generalization to the exterior of $D$, the fact that $\operatorname{div}\left(\vec{u}^{+}\right)$and $\operatorname{div}\left(\vec{u}^{-}\right)$are harmonic functions on their domains of definition, and arguments similar to those we used in Theorem 3 , we have $\vec{u}=\vec{v}$ on $\mathbf{R}^{n}$. From this representation formula for $\vec{u}$, Lemma 1, (1.5), and the estimate $\|N(\nabla \vec{w})\|_{L^{2}(\partial D)} \leq C\left\|\vec{h}_{1}\right\|_{L_{1}^{2}(\partial D)}$ (see $\left.[\mathrm{D}, \mathrm{K}, \mathrm{V}, 2]\right)$ we get

$$
\begin{aligned}
\left\|\nabla \vec{u}^{ \pm}\right\|_{L^{2}(\partial D)} \leq C\left[\left\|\vec{h}_{1}\right\|_{L_{1}^{2}(\partial D)}+\left\|\vec{h}_{2}\right\|_{L^{2}(\partial D)}\right. \\
\left.+\left\|\operatorname{div}\left(\vec{u}^{+}\right)\right\|_{L^{2}(\partial D)}+\left\|\operatorname{div}\left(\vec{u}^{-}\right)\right\|_{L^{2}(\partial D)}\right] .
\end{aligned}
$$

From (3.22), (3.21), and (3.20) we get (3.3).

Finally, as in Theorem 2, the estimate (3.2) and the fact that the operator in Theorem 4 is invertible when $D$ is smooth (this can be proved with arguments similar to those in Theorem 2) imply Theorem 4. We will leave these details as an easy exercise for the reader.

Remark 3.1. In this case, and for analogous reasons to those given in Remark 2.1 there is also an $\varepsilon=\varepsilon(D)$ and a constant $C$, so that when $u$ is as in Theorem 3 the following estimate holds:

$$
\left\|N\left(\nabla \vec{u}^{ \pm}\right)\right\|_{L^{2+\varepsilon}(\partial D)} \leq C\|u\|_{W^{1,2}(B)} .
$$

Remark 3.2. A more careful analysis of the identity (3.17) shows that Theorem 3 also holds under the assumptions $2 \mu+k \lambda>2 \tilde{\mu}+k \tilde{\lambda}$ for all $k=2, \ldots, n$, and $\mu>\tilde{\mu}$. 


\section{TRANSMISSION FOR PARABOLIC EQUATIONS}

In this section we will show that the methods outlined before also apply to obtain regularity properties for solutions to parabolic equations with transmission conditons. In particular, and for the sake of simplifying we will only consider the operator $D_{t} u-\operatorname{div}(A(X, t) \nabla u(X, t))$ on $B \times[0, T], T>0$, where $A(X, t)=k I \chi_{\Omega_{T}}+I \chi_{\mathbf{R}^{n+1} \backslash \Omega_{T}}, \Omega_{T}$ is a Lipschitz domain in time and space contained in $B_{1 / 2} \times[-\infty, T]$ whose lateral boundary is not horizontal, and $I$ is the identity matrix. In this case we have the following theorem.

Theorem 6. Let $u \in C\left([0, T], W^{1 / 2}(B)\right) \cap L^{2}\left([0, T], W^{1,2}(B)\right)$ be a weak solution to the above parabolic operator on $B \times[0, T]$ with $u(X, 0)=0$ on $B$. Assume that the lateral boundary of $\Omega_{T}$ is given by $\Gamma=\{(X, t) \mid t=\varphi(X), t \leq T\}$, so that the interior of $\Omega_{T}$ is the region $\Omega_{T}^{+}=\{(X, t) \mid t>\varphi(X), t \leq T\} \subset B_{1 / 2} \times$ $[-\infty, T]$, and the exterior the complement $\Omega_{T}^{-}=\{(X, t) \mid t<\varphi(X), t \leq T\}$, where $\varphi$ is a Lipschitz function for which there is a positive number $\gamma$ such that $\gamma^{-1} \leq|\nabla \varphi(X)|$ and $\varphi(0)<0$. We will also assume the following conditions:

a. For each $t \leq T$ the level set $\Gamma_{t}=\left\{P \in \mathbf{R}^{n} \mid \varphi(P)=t\right\}$ is the boundary of a bounded connected Lipschitz domain $D_{t}$ in $\mathbf{R}^{n}$, such that when $0 \leq t \leq T$, their Lipschitz character is uniformly controlled and their diameters are proportional.

b. There exist numbers $\alpha$ and $r_{0}$ in $(0,1)$ such that for each $(Q, t) \in \partial D_{t}$, $0 \leq t \leq T$, and $X \in \Gamma^{+}(Q)$ with $|X-Q|=r \leq r_{0}$ (respectively $X \in \Gamma^{-}(Q)$ ) the cylinder $B_{2 \alpha r}(X) \times\left[t-4(\alpha r)^{2}, t\right]$ is contained in $\Omega_{T}^{+}$(respectively in $\Omega_{T}^{-}$). Also, for each $(Q, t) \in \partial D_{t}$ with $0 \leq t \leq T$, the surface measure on $\partial \Omega_{T}$ of the set $B_{r}(Q) \times\left[t-r^{2}, t\right) \cap \partial \Omega_{T}$ is bounded from above and below by $\alpha s^{n+1}$ and $\alpha^{-1} s^{n+1}$ for all $r \leq r_{0}$.

Then there is a compact subset $K \subset B \times[0, T]$ away from the lateral boundary of $\Omega_{T}$ and a constant $C$ depending on $\gamma, \alpha, T$, and the Lipschitz character of the domains $D_{t}$, such that

$$
\begin{gathered}
\left\|N\left(\nabla u^{ \pm}\right)\right\|_{L^{2}\left(\partial \Omega_{T} \cap B \times[0, T]\right)}+\left\|D_{t} u\right\|_{L^{2}\left(B_{3 / 4} \times[0, T]\right)} \\
\leq C\left\{\|u\|_{L^{2}\left([0, T], W^{1.2(B))}\right.}+\left\|D_{t} u\right\|_{L^{2}(K)}\right\} .
\end{gathered}
$$

Proof. As usual, to show the above estimate we might assume that the spatial and time derivatives of $u$ are bounded continuous functions at both sides of $\Omega_{T}$ (which is the case when the boundary of $\Omega_{T}$ is smooth (see [L, R, U]), and standard methods will give the above estimate under the conditions of Theorem 6.

Let $u$ be as in Theorem 6 , then $u$ satisfies

$$
\int_{0}^{T} \int u D_{t} \phi-\left\langle\left(k I \chi_{\Omega_{T}}+I \chi_{\mathbf{R}^{n+1} \backslash \Omega_{T}}\right) \nabla u, \nabla \phi\right\rangle d X d t=0
$$

for all $\phi \in C_{0}^{\infty}(B \times[0, T))$.

Observe that the above weak formulation and the continuity of $u$ across the lateral boundary of $\Omega_{T}$ imply that $D_{t} u-k \quad \Delta u=0$ on $\Omega_{T}, D_{t} u-\Delta u=0$ on $B \times[0, T] \backslash \Omega_{T}$, and $k\left\langle\nabla u^{+}, N_{t}\right\rangle=\left\langle\nabla u^{-}, N_{t}\right\rangle$ when $\varphi(Q)=t, 0 \leq t \leq T$, $Q \in B_{1 / 2}$, and where $N_{t}$ is the exterior unit normal to $D_{t}$ at $(Q, t) \in \partial D_{t}$. 
We have the following estimate:

$$
\begin{aligned}
& \int_{0}^{T} \int_{B_{3 / 4}}\left(D_{t} u\right)^{2} d X d t \\
& \quad \leq C\left[\int_{0}^{T} \int_{\partial D_{t}}\left[\left|\nabla u^{+}\right|^{2}+\left|\nabla u^{-}\right|^{2}\right] d \sigma_{t} d t+\int_{0}^{T} \int_{B}|\nabla u|^{2} d X d t\right] .
\end{aligned}
$$

To see this, let $\psi \in C_{0}^{\infty}(B)$ with $\psi=1$ on $B_{3 / 4}$ and $\psi=0$ outside $B_{7 / 8}$. Then integration by parts gives

$$
\begin{aligned}
\int_{0}^{T} \int & \left(D_{t} u\right)^{2} \psi^{2} d X d t \\
= & \int_{0}^{T} \int D_{t} u\left(k \chi_{\Omega_{T}}+\chi_{\mathbf{R}^{n+1} \backslash \Omega_{T}}\right) \Delta u \psi^{2} d X d t \\
= & \int_{0}^{T} \int_{\partial D_{t}}\left[k\left\langle\nabla u^{+}, N_{t}\right\rangle D_{t} u^{+}-\left\langle\nabla u^{-}, N_{t}\right\rangle D_{t} u^{-}\right] \psi^{2} d \sigma_{t} d t \\
& -\frac{1}{2} \int_{0}^{T} \int\left(k \chi_{\Omega_{T}}+\chi_{\mathbf{R}^{n+1} \backslash \Omega_{T}}\right) D_{t}\left(|\nabla u|^{2}\right) \psi^{2} d X d t \\
& -2 \int_{0}^{T} \int\left(k \chi_{\Omega_{T}}+\chi_{\mathbf{R}^{n+1} \backslash \Omega_{T}}\right) D_{t} u \psi \nabla u \nabla \psi d X d t
\end{aligned}
$$

From the fact that $u$ is continuous across the lateral boundary of $\Omega_{T}$, the tangential derivatives of $u^{+}$and $u^{-}$should coincide across this boundary. Thus, $D_{i} u^{+}+D_{t} u^{+} D_{i} \varphi=D_{i} u^{-}+D_{t} u^{-} D_{i} \varphi$ when $t=\varphi(X)$ and $i=1, \ldots, n$, which implies $\left\langle\nabla u^{+}-\nabla u^{-}, \nabla \varphi\right\rangle=\left(D_{t} u^{+}-D_{t} u^{-}\right)|\nabla \varphi|^{2}$ for $t=\varphi(X)$. Hence, $\left|D_{t} u^{+}-D_{t} u^{-}\right| \leq C\left|\nabla u^{+}-\nabla u^{-}\right|$on the lateral boundary of $\Omega_{T}$. On the other hand, the integrand in the second integral on the right-hand side of (4.2) equals $\left[k\left\langle\nabla u^{+}, N_{t}\right\rangle\left(D_{t} u^{+}-D_{t} u^{-}\right)\right] \psi^{2}$, and the third integral is larger than

$$
\frac{1}{2} \int_{\{0 \leq \varphi(X) \leq T\}}\left(\left|\nabla u^{-}\right|^{2}-k\left|\nabla u^{+}\right|^{2}\right)(X, \varphi(X)) \psi^{2}(X) d X
$$

Using the co-area formula $[\mathrm{M}]$ this integral equals

$$
\frac{1}{2} \int_{0}^{T} \int_{\partial D_{t}}\left(\left|\nabla u^{-}\right|^{2}-k\left|\nabla u^{+}\right|^{2}\right) \psi^{2}|\nabla \varphi|^{-1} d \sigma_{t} d t .
$$

From all these and Schwartz's inequality we get (4.1).

Defining for each time $t$ the function

$$
w(X, t)=u(X, t)-\Pi\left(D_{t} u(\cdot, t) \chi_{B_{3 / 4}}\right)(X)+v^{t}(X),
$$

where the second term denotes the Newtonian potential of $D_{t} u(\cdot, t) \chi_{B_{3 / 4}}$, and $v^{t} \in W^{1,2}\left(B_{3 / 4}\right)$ the unique weak solution to $\operatorname{div}\left(\left(k I \chi_{D_{l}}+I \chi_{\mathbf{R}^{n} \backslash D_{l}}\right) \nabla v^{t}(X)\right)=$ $F_{t}, v^{t}=0$ on $\partial B_{3 / 4}$, where $F_{t}$ denotes the linear functional given by

$$
F_{t}(\phi)=\int_{\partial D_{t}}(k-1)\left\langle\nabla \Pi\left(D_{t} u(\cdot, t) \chi_{B_{3 / 8}}\right), N_{t}\right\rangle \phi d \sigma_{t},
$$

it turns out that $w(\cdot, t)$ is a weak solution to $\operatorname{div}\left(\left(k I \chi_{D_{t}}+I \chi_{\mathbf{R}^{n} \backslash D_{t}}\right) \nabla u(X)\right)=0$ on $B_{3 / 4}$. From Theorem 1 and the obvious generalization of this theorem we 
get for all $0 \leq t \leq T$

$$
\begin{aligned}
\left\|N\left(\nabla u^{ \pm}(\cdot, t)\right)\right\|_{L^{2}\left(\partial D_{t}\right)} \leq C\{\| & u(\cdot, t) \|_{W^{1 / 2}\left(B_{3 / 4}\right)} \\
& +\left\|N\left(\nabla \Pi^{ \pm}\left(D_{t} u(\cdot, t) \chi_{B_{3 / 4}}\right)\right)\right\|_{L^{2}\left(\partial D_{t}\right)} \\
& \left.+\left\|\Pi\left(D_{t} u(\cdot, t) \chi_{\left.B_{3 / 4}\right)}\right)\right\|_{W^{1,2}\left(B_{3 / 4}\right)}\right\} .
\end{aligned}
$$

On the other hand, we will show that there is a number $1<p<2$ such that

$$
\left\{\int_{0}^{T} \int_{\partial D_{t}}\left|N\left(\nabla \Pi^{ \pm}\left(D_{t} u(\cdot, t) \chi_{B_{3 / 4}}\right)\right)\right|^{2} d \sigma_{t} d t\right\}^{1 / 2} \leq C\left\|D_{t} u\right\|_{L^{p}\left(B_{3 / 4} \times[0, T]\right)}
$$

and

(4.5) $\left\|\Pi\left(D_{t} u(\cdot, t) \chi_{B_{3 / 4}}\right)\right\|_{W^{1,2}\left(B_{3 / 4}\right)} \leq C\left\|D_{t} u(\cdot, t)\right\|_{L^{p}\left(B_{3 / 4}\right)} \quad$ for all $0 \leq t \leq T$,

where $C$ is as in the statement of this theorem. Squaring both sides of (4.3), integrating in time from 0 to $T$, and using Hölder's inequality together with (4.4) and (4.5) we obtain

$$
\begin{aligned}
& \left\|N\left(\nabla u^{ \pm}\right)\right\|_{L^{2}\left(\partial \Omega_{T} \cap B \times[0, T]\right)} \\
& \quad \leq C\left\{\|u\|_{L^{2}\left([0, T], W^{1,2}(B)\right)}+\left\|D_{t} u\right\|_{L^{2}\left(K_{\varepsilon}\right)}+\varepsilon\left\|D_{t} u\right\|_{L^{2}\left(B_{3 / 4} \times[0, T]\right)}\right\},
\end{aligned}
$$

where $\varepsilon>0$ is any positive number and $K_{\varepsilon}$ is a compact subset of $B \times$ $[0, T] \backslash \Omega_{T}$. Choosing $\varepsilon>0$ sufficiently small we get from (4.1)

$$
\begin{gathered}
\left\|N\left(\nabla u^{ \pm}\right)\right\|_{L^{2}\left(\partial \Omega_{T} \cap B \times[0, T]\right)}+\left\|D_{t} u\right\|_{L^{2}\left(B_{3 / 4} \times[0, T]\right)} \\
\leq C\left\{\|u\|_{L^{2}\left([0, T], W^{1,2}(B)\right)}+\left\|D_{t} u\right\|_{L^{2}(K)}\right\},
\end{gathered}
$$

where $K$ is a compact subset of $B \times[0, T]$ away from the lateral boundary of $\Omega_{T}$.

Inequality (4.5) follows from fractional integration [St]. To show (4.4), we observe that for $(Q, t) \in \partial D_{t}$ and $(X, t) \in \Gamma^{+}(Q)$ with $r=|X-Q|$, $\left|\nabla \Pi\left(D_{t} u(\cdot, t) \chi_{B_{3 / 4}}\right)(X)\right|$ is bounded by

$$
\begin{aligned}
& \int_{B_{3 / 4}}|X-Y|^{1-n}\left|D_{t} u(Y, t)\right| d Y \\
& \quad \leq C\left[\int_{B_{3 / 4} \backslash B_{\alpha r}(X)}|Q-Y|^{1-n}\left|D_{t} u(Y, t)\right| d Y+r \sup _{B_{\alpha r}(X)}\left|D_{t} u(Y, t)\right|\right] .
\end{aligned}
$$

The first term on the right-hand side is bounded by

$$
I_{1}(Q, t)=\sup _{0<s<1}\left[\frac{1}{\left|B_{s}(Q)\right|^{1-\beta / n}} \int_{B_{s}(Q)}\left|D_{t} u(Y, t)\right|^{p} \chi_{B_{3 / 4}} d Y\right]^{1 / p},
$$

when $1<\beta<p$.

On the other hand, since $\varphi(0)<0$ we might extend $u$ as zero for $t<0$ as a solution to the parabolic operator inside $\Omega_{T}$ on a cylindrical neighborhood below the set $D_{0} \times\{0\}$. From well-known interior estimates we have

$$
\begin{aligned}
r \operatorname{Sup}_{B_{\alpha r}(X)}\left|D_{t} u(Y, t)\right| & \leq C \frac{1}{r^{n+1}} \int_{B_{2 \alpha r}(X)} \int_{t-4(\alpha r)^{2}}^{t}\left|D_{t} u \chi_{B_{3 / 4}}\right| d Y d s \\
& \leq C\left\{\frac{1}{r^{n+2-p}} \int_{B_{2 r}(Q)} \int_{t-4 r^{2}}^{t}\left|D_{t} u \chi_{B_{3 / 4}}\right|^{p} d Y d s\right\}^{1 / p},
\end{aligned}
$$


and if $I_{2}(Q, t)$ denotes the supremum for $0<r<1$ of the above integrals we have

$$
N\left(\nabla \Pi^{+}\left(D_{t} u(\cdot, t) \chi_{B_{3 / 4}}\right)\right)(Q, t) \leq C\left[I_{1}(Q, t)+I_{2}(Q, t)\right],
$$

and a simple Vitali covering argument [St] shows that

$$
\left\|I_{1}(\cdot, t)\right\|_{L^{2}\left(\partial D_{t}\right)} \leq C\left\|D_{t} u(\cdot, t)\right\|_{L^{p}\left(B_{3 / 4}\right)}
$$

for $2<p(n-1) /(n-\beta)$. Also a parabolic Vitali covering argument and condition $\mathrm{b}$ in Theorem 5 gives $\left\|I_{2}\right\|_{L^{2}\left(\partial \Omega_{T}\right)} \leq C\left\|D_{t} u\right\|_{L^{p}\left(B_{3 / 4} \times[0, T]\right)}$ for $p>$ $2(n+2) /(n+3)$. A similar argument gives similar estimates for the exterior nontangential maximal function of $\nabla \Pi\left(D_{t} u(\cdot, t) \chi_{B_{3 / 4}}\right)$. Choosing $\beta$ with $1<$ $\beta<p$, and $p>\max \{2(n+2) /(n+3), 2(n-\beta) /(n-1)\}$ proves the claim and completes the proof of this theorem.

Remark 4.1. The reader will observe that when $\Omega_{T}$ has the shape of a cone $(\varphi(0)=0$, and $0 \leq \varphi)$, condition $\mathrm{b}$ in Theorem 6 can only hold uniformly for points $(Q, t) \in \partial D_{t}$ with $0<\varepsilon \leq t \leq T$. But even in this case, the argument in Theorem 6 and the estimate

$$
\|\nabla \Pi(f)\|_{L^{2}\left(\partial D_{t}\right)} \leq C\|f\|_{L^{p}\left(B_{3 / 4}\right)} \text { for } 2<p \frac{n-1}{n-\beta} \text { and } f \in L^{p}\left(\partial D_{t}\right)
$$

(this follows again from a Vitali covering argument) show that

$\left\|\nabla u^{ \pm}\right\|_{L^{2}\left(\partial \Omega_{T} \cap B \times[0, T]\right)}+\left\|D_{t} u\right\|_{L^{2}\left(B_{3 / 4} \times[0, T]\right)} \leq C\left\{\|u\|_{L^{2}\left([0, T], W^{1.2}(B)\right)}+\left\|D_{t} u\right\|_{L^{2}(K)}\right\}$,

where $C$ depends only on the uniform Lipschitz character of the domains $D_{t}$, $0<t \leq T$. In this case, for the nontangential maximal functions, which should be defined with respect to cones fitting the domains $D_{t}, 0<t \leq T$ (the heights of this cone should decrease with $t \in(0, T])$, we get

$$
\begin{gathered}
\left.\left\|N\left(\nabla u^{ \pm}\right)\right\|\right)_{L^{2}\left(\partial \Omega_{T} \cap B \times[\varepsilon, T]\right)}+\left\|D_{t} u\right\|_{L^{2}\left(B_{3 / 4} \times[0, T]\right)} \\
\leq C\left\{\|u\|_{L^{2}\left([0, T], W^{1.2(B))}\right.}+\left\|D_{t} u\right\|_{L^{2}(K)}\right\},
\end{gathered}
$$

where now $C$ depends on the Lipschitz character of the domains $D_{t}$ and $\varepsilon>0$.

\section{REFERENCES}

[C,Mc,Me] R. R. Coifman, A. McIntosh, and Y. Meyer, L'intégrale de Cauchy définit un opérateur borné sur $L^{2}$ pour les courbes lipschitziennes, Ann. of Math. (2) 116 (1982), 361-387.

[C,Z] A. P. Calderon and A. Zygmund, On singular integrals, Amer. J. Math. 78 (1956), 289-309.

[D,K,V,1] B. E. J. Dahlberg, C. E. Kenig, and G. C. Verchota, The Dirichlet problem for the biharmonic equation in a Lipschitz domain, Ann. Inst. Fourier (Grenoble) 36 (1986), 109-135.

$[\mathrm{D}, \mathrm{K}, \mathrm{V}, 2] \ldots$, Boundary value problems for the systems of elastostatics in Lipschitz domains, Duke Math. J.,

[E,F,V] L. Escauriaza, E. B. Fabes, and G. Verchota, On a regularity theorem for weak solutions to transmission problems with internal Lipschitz boundaries, Proc. Amer. Math. Soc. (to appear).

[F,J,R] E. B. Fabes, M. Jodeit, and N. M. Riviére, Potential techniques for boundary value problems on $C^{1}$ domains, Acta Math. 141 (1978), 165-186.

[F] K. O. Friedrichs, On the boundary value problems of the theory of elasticity and Korn's inequality, Ann. of Math. (2) 48 (1947), 441-471.

[G] M. Giaquinta, Multiple integrals in the calculus of variations and non-linear elliptic systems, Ann. of Math. Stud., No. 105, Princeton Univ. Press, Princeton, N.J., 1983. 
[J,K,1] D. S. Jerison and C. E. Kenig, The Neumann problem on Lipschitz domains, Bull. Amer. Math. Soc. 4 (1981), 203-207.

[J,K,2] __, Boundary value problems on Lipschitz domains, MAA Stud. Math., vol. 23, Studies in Partial Differential Equations (W. Littman, ed.), 1982, pp. 1-68.

[K] C. E. Kenig, Hardy spaces and the Neumann problem in $L^{p}$ for Laplace's equation in Lipschitz domains, Ann. of Math. (2) 125 (1987), 437-465.

[L,R,U] O. A. Ladyzenskaja, V. Ja. Rivkind, and N. N. Ural'ceva, The classical solvability of diffraction problems, Proc. Steklov. Inst. Math. 92 (1966), 132-166.

[M] V. G. Maziâ, Sobolev spaces, Springer-Verlag, New York and Berlin, 1985.

[N] J. Necas, Les méthods directes en théorie des équations élliptiques, Academia, Prague, 1967.

[S,W] J. Serrin and H. Weinberger, Isolated singularities of solutions of linear elliptic equations, Amer. J. Math. 88 (1966), 258-272.

[St] E. M. Stein, Singular integrals and differentiability properties of functions, Princeton Univ. Press, Princeton, N.J., 1970.

[V] G. C. Verchota, Layer potentials and boundary value problems for Laplace's equation in Lipschitz domains, J. Funct. Anal. 59 (1984), 572-611.

Department of Mathematics, University of Chicago, Chicago, Illinois 60637

Current address: Consejo Superior Investigaciones Cientificas, Serrano 11728006 Madrid, Spain

E-mail address: escaurza@math.uchigo.edu

School of Mathematics, University of Minnesota, Minneapolis, Minnesota 55455

Current address: Postech, Pohaug, Institute of Science and Technology P. O. Box 125, Pohaug, 790-600 Korea

E-mail address: seoj@posmath.postech.ac.kr 